Review article

\title{
The role of norepinephrine in the pathophysiology of schizophrenia
}

\author{
Verónica Mäki-Marttunen $^{\mathrm{a}, *}$, Ole A. Andreassen ${ }^{\mathrm{b}}$, Thomas Espeseth ${ }^{\mathrm{a}, \mathrm{c}}$ \\ ${ }^{a}$ Department of Psychology, University of Oslo, Postboks 1094, Blindern, 0317 Oslo, Norway \\ ${ }^{\mathrm{b}}$ CoE NORMENT, KG Jebsen Centre for Psychosis Research, Division of Mental Health and Addiction, Oslo University Hospital, Building 49, P.O. Box 4956 Nydalen, N- \\ 0424 Oslo, Norway \\ ${ }^{\mathrm{c}}$ Bjørknes College, Lovisenberggata 13, 0456 Oslo, Norway
}

\section{A R T I C L E I N F O}

\section{Keywords:}

Schizophrenia

Locus coeruleus

Norepinephrine

Dopamine

Cognition

\begin{abstract}
A B S T R A C T
Several lines of evidence have suggested for decades a role for norepinephrine (NE) in the pathophysiology and treatment of schizophrenia. Recent experimental findings reveal anatomical and physiological properties of the locus coeruleus-norepinephrine (LC-NE) system and its involvement in brain function and cognition. Here, we integrate these two lines of evidence. First, we review the functional and structural properties of the LC-NE system and its impact on functional brain networks, cognition, and stress, with special emphasis on recent experimental and theoretical advances. Subsequently, we present an update about the role of LC-associated functions for the pathophysiology of schizophrenia, focusing on the cognitive and motivational deficits. We propose that schizophrenia phenomenology, in particular cognitive symptoms, may be explained by an abnormal interaction between genetic susceptibility and stress-initiated LC-NE dysfunction. This in turn, leads to imbalance between LC activity modes, dysfunctional regulation of brain network integration and neural gain, and deficits in cognitive functions. Finally, we suggest how recent development of experimental approaches can be used to characterize LC function in schizophrenia.
\end{abstract}

\section{Introduction}

Schizophrenia (SCZ) is a severe mental disorder with a lifetime risk of about $1 \%$, characterized by hallucinations and delusions, lack of motivation and blunted affect, and cognitive deficits (Cardno and Gottesman, 2000; McGrath et al., 2008; Owen et al., 2016; Sullivan et al., 2003). Psychosis is the cardinal trait of SCZ, characterized by positive symptoms that involve a loss of contact with reality: hallucinations, delusions and disorganized thoughts (Owen et al., 2016). These are often accompanied by negative (deficit) symptoms such as blunted emotions, apathy, lack of interest, and by disorganized behavior. Despite a variation in clinical presentation, the most common picture in SCZ is paranoid delusions and auditory hallucinations with onset late in adolescence or in early adulthood, and reduced quality of life (Owen et al., 2016). These manifestations of the disorder have changed little since the description of SCZ as "dementia praecox" more than a century ago (Kraepelin, 1893). Sustained recovery occurs in less than $14 \%$ within the first five years following a psychotic episode, and is only marginally better after 25 years (16\%) (Jaaskelainen et al., 2013; Lieberman and First, 2018). In Europe, less than $20 \%$ of people with SCZ are employed, and in the US nearly $20 \%$ of them are homeless (Folsom et al., 2005; Marwaha, 2007), with high global impact on
Disability-Adjusted Life Years (DALY) measures (Rossler et al., 2005). Due to the serious medical and socio-economic consequences of this condition, the understanding of SCZ is recognized world-wide as a pressing need.

Cognitive abnormalities and motivational impairment are key components of SCZ, and unlike positive symptoms, cognitive dysfunction predate diagnosis, predict functional outcome, resist treatment, and often persist throughout life (Nuechterlein et al., 2012). Cognitive dysfunction often co-occur with motivational impairment, and have devastating consequences for patients, and there is no effective treatment. SCZ is highly heritable (about $80 \%$ ), and cognitive phenotypes and SCZ are significantly correlated. This covariance is due to genetic factors as shown in twin studies (Owens et al., 2011; Toulopoulou et al., 2007) and genome-wide association studies (Smeland and Andreassen, 2018; Smeland et al., 2019, 2017). Polygenetic signals associated with cognitive function have been shown to be associated with risk of SCZ (Fernandes et al., 2013; Lencz et al., 2014; Richards et al., 2019). In addition to genetic factors, environmental factors, such as those related to pregnancy and birth, diet, migration, substance use, socioeconomic factors, and stressful experiences during adolescence, play a central role and can precipitate the onset of the disease (Owen et al., 2016). However, the mechanisms underlying SCZ are still not identified. To

\footnotetext{
* Corresponding author at: Postboks 1094, Blindern, 0317, Oslo, Norway.

E-mail address: makimarttunen.veronica@gmail.com (V. Mäki-Marttunen).
} 
gain a clearer insight into the pathophysiology of the disorder, an important strategy is to investigate cognitive and motivational dysfunctions (Insel, 2010). Although these impairments have been associated with SCZ for a long time (Bleuler, 1950), the mechanistic causes are still not clearly understood.

Researchers have long accepted that the symptomatology of SCZ partly emerges from disrupted neuromodulation, as evidenced by the dopamine (DA) theory that has been around for the past decades (Carlsson, 1978; Davis, 1991), and later serotonin and glutamate theories (Aghajanian and Marek, 2000; Eggers, 2013; Howes, 2015). Recent genome-wide association studies support the hypothesis that risk of schizophrenia is related to abnormal modulation or regulation of neurotransmission (Devor et al., 2017). The abnormal modulation of brain processing in SCZ has been mainly attributed to DA. Notably, a major neuromodulator of brain function and neural gain, norepinephrine (NE), and its interaction with DA, have received little attention in the characterization of this disease.

A few decades ago, a DA-NE hypothesis of SCZ emerged as an attempt to explain symptoms and observations that could not be accounted for by DA dysregulation only (Stein and Wise, 1971; Van Kammen and Kelley, 1991). Indeed, van Kammen and Kelley (1991) claimed that "pharmacological evidence supporting a role of NE in psychosis is almost as strong as for DA", after the observation that DA agonists that have effects on psychosis have also strong effects on NE system. Since then, relatively little experimental work has further explored the integrity of NE system in SCZ. This may be partly due to the serious limitations in measuring the activity of this system in vivo in humans. Recent reviews of pharmacological evidence (Borodovitsyna et al., 2017; Fitzgerald, 2014; Yamamoto et al., 2014) have renewed the interest on NE system in SCZ, suggesting that an altered transmission is linked to the etiology of the disease.

In parallel, in the past years, increased focus has been paid to the locus coeruleus-norepinephrine (LC-NE) system in SCZ pathophysiology because of the growing evidence for its role in facilitating motivation and cognition. The LC-NE system and its role in attention and attentional control have been reviewed extensively elsewhere (Aston-Jones and Cohen, 2005; Aston-Jones and Waterhouse, 2016; Sara, 2009; Unsworth and Robison, 2017). Furthermore, this system has been linked to several mental illnesses that show cognitive deficits, such as dementia and attention deficit hyperactivity disorder (ADHD) (Castellanos and Tannock, 2002; Jacobs et al., 2015; Ressler and Nemeroff, 2001). LC shows selective degeneration in dementia, Alzheimer's disease and Parkinson's disease, and even during normal ageing (Braak et al., 2011; Gesi et al., 2000; McMillan et al., 2011; Szot et al., 2010; Weinshenker, 2008). All these conditions present cognitive deficits that arise from a dysregulation of prefrontal signaling (Arnsten et al., 2012). Despite the nature of these conditions is very different, their associated cognitive symptoms have been linked to the LC-NE system (for a review, see Borodovitsyna et al., 2017 and citations therein). In this review, we first describe the main characteristics of the LC-NE system and how they relate to brain function and cognition, including recent findings that render this system as more complex and versatile than originally thought. We then review how the LC-NE system may connect to the pervasive cognitive and motivational deficits observed in SCZ. At the end, we suggest open questions and experimental approaches to advance our knowledge about LC function in SCZ.

\section{The locus coeruleus-norepinephrine system in the healthy brain}

\subsection{Overview of the LC-NE system}

\subsubsection{Structure and function of the LC-NE system}

The locus coeruleus is a small nucleus of cells located in the lateral wall of the brainstem by the fourth ventricle. The LC contains about 20.000 neurons in humans and produces most of the NE released in the

\section{Locus ceruleus projections}

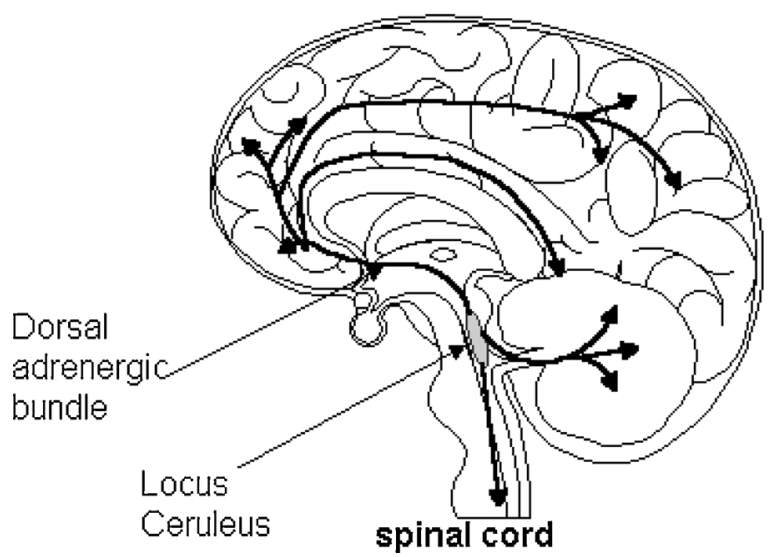

Fig. 1. LC projections. The locus coeruleus is the primary source of NE to the mammalian cortex.

brain. Different from other neuromodulatory systems, the NE system is highly centralized because most of the noradrenergic neurons of the brain are contained in the LC. At the same time, the LC projects vastly to most of the brain, making both synaptic and non-synaptic contacts (Fig. 1; see Aston-Jones and Waterhouse, 2016, for a review). The LC projects to subcortical areas such as the olfactory bulb and dorsal hippocampus; cortical areas, in particular the prefrontal cortex (PFC); and reciprocally to other neuromodulatory systems such as the cholinergic, DA-ergic and serotoninergic systems. In turn, LC receives direct input from the cortex and several subcortical areas including amygdala and hypothalamus, and from giganto- and paragiganto-cellularis nuclei (Szabadi, 2013). NE exerts its effects through noradrenergic receptors (alpha-1, alpha-2 and beta; see Section 2.1.2), which are found over the whole cortex. In addition to NE, LC neurons release galanin (Weinshenker and Holmes, 2016) and DA (see Section 2.1.4). In summary, LC can be considered a hub that projects to most of the brain, integrates information from sympathetic, parasympathetic, limbic and cortical centers, and is connected to other neuromodulatory systems.

Despite the small size and phylogenetically early origin of the LC, its neuronal organization is quite complex and diverse (as reviewed by Schwarz and Luo, 2015). The traditional view is that LC neuronal projections to the cortex are uniform across the cortex and that LC neurons fire synchronously through gap junctions. Three main lines of findings challenge this view and suggest that the activity in LC may be more specific than originally thought.

First, work in rats adds evidence on a specialization in the projections from LC to cortical regions. A study using genetic markers of development have identified subpopulations within the LC and across the NE-releasing nuclei: four subpopulations that share genetic markers show specificity in their projection patterns, nucleus localization and axon morphology (Robertson et al., 2013). Furthermore, the density of $\mathrm{NE}$ varicosities (i.e. axonal buttons) varies across the cortex, with more dense end points in prefrontal regions than in sensory motor and thalamic regions (Agster et al., 2013). Using new viral-genetic techniques used in neuroanatomy studies, where viral libraries expressing RNA barcodes are transduced into neuron populations, Kebschull et al., 2016 showed that some LC neurons project to one single brain area, whereas others projects to many areas. In addition, a subpopulation of neurons from the LC project preferentially to the motor cortex, and another subpopulation projects preferentially to sections of the PFC with little target overlap (medial PFC, ACC and OBF; Chandler et al., 2013; Chandler and Waterhouse, 2012). These subpopulations of neurons have different properties, namely glutamate-related excitability and expression patterns of synaptic proteins (Chandler et al., 2014a). A hypothesized functional relevance of these different properties is that 
during quiet wakefulness, the PFC is at the optimal level of neural excitability driven by LC for performing attentional tasks, while the motor cortex is at a suboptimal level. During stress-evoked increase in LC firing, PFC switches to a suboptimal state for mental operations, while motor cortex switches to an optimal level for motor behavior (Chandler et al., 2014a). This is in agreement with the observed effects of stress: suboptimal performance but enhanced motor ("fight and flight") responsivity (see Section 2.1.3). In sum, LC projections to the cortex are far from homogeneous, and this may be key for LC modulation of cortical activity.

Second, although LC neurons are at least partially coupled through gap junctions, recent data suggest that synchrony between LC cells is rare (Totah et al., 2018). Totah et al. describe two physiologically distinct subtype of neurons interspersed in the nuclei ("wide" and "narrow" units) that constitute ensembles uncorrelated with each other, most likely because they exert mutual lateral inhibition. This has two implications: a) neuromodulation is targeted, because units connected through gap junctions project to the same target in the forebrain; and $b$ ) afferents to LC targeting different ensembles/unit types could have specific effects, for example generating a disproportionate desynchronization of the nucleus if targeting wide units. Behaviorally relevant heterogeneity of function in LC has been further supported by work showing that subpopulations of cells with specific efferent projections are activated differentially during Pavlovian fear conditioning and extinction, despite all of them responding globally to aversive stimuli (Uematsu et al., 2017).

Third, the firing of LC neurons oscillates at infra-slow frequencies (0.09 and $0.4-0.5 \mathrm{~Hz}$, Totah et al., 2018). Strikingly, their firing is phase-locked to slow cortical oscillations, always occurring during the down-to-up phase (although not every neuron may fire in every cycle). Slow oscillations are found in many measures of brain activity, including local field potentials (LFP), electroencephalography (EEG) and functional magnetic resonance imaging (fMRI), and are thought to underlie large-scale communication within brain networks. These findings place the LC at the core of the mechanisms that drive the switching between the functional networks that underlie different brain processes (Section 2.2.2).

Although the reviewed studies have been done in rodents, it is expected that the human LC presents a similar functional organization. The impact of this specificity on behavior and brain modulation is yet to be further explored, and new research avenues will further explain the complexity of behaviors that implicate the NE system. Altogether, the findings suggest that LC neurons are more specific in terms of projections, constitution and activation than originally thought. These attributes ascribe specific functional roles to LC regarding its arousing effect on the cortex (Chandler et al., 2019).

\subsubsection{NE receptors}

The action of NE on its cortical and subcortical targets is mediated by different types of receptors and autoreceptors. NE has two receptor types - alpha and beta adrenoreceptors - and several subtypes, which have different affinity, dynamics and actions in the brain (Table 1; for reviews: Chamberlain et al., 2006; Coull, 1994). Adrenergic receptors belong to the family of receptors that are coupled to G-protein, and each receptor activates a specific intracellular cascade (Table 1, Lodish et al., 2000). The receptors are present in all types of cells (pyramidal cells, interneurons and glial cells, O'Donnell et al., 2012) with an exquisite arrangement of expression profiles according to cell type and subtype, part of the cell body, and layer, thus multiplying the variety and complexity of effects of NE in the brain (O'Donnell et al., 2012). Alpha-1 adrenoreceptors are the most prevalent in the brain; alpha- 2 receptors are less ubiquitous. Presynaptic alpha-2 receptors are present in noradrenergic terminals (autoreceptors) as well as in serotoninergic, dopaminergic and glutamatergic neurons (Jimenez-Rivera et al., 2012; Millan et al., 2000; Scheibner et al., 2001; Shields et al., 2009). Postsynaptic alpha-2 receptors have excitatory effects on pyramidal cells of the prefrontal cortex. The alpha- 2 receptors have the highest affinity for $\mathrm{NE}$, and postsynaptic alpha- 2 receptors have been related to improved prefrontal cortex and hippocampal function. Indeed, stimulation of alpha- $2_{\mathrm{A}}$ receptors is related to strengthened delay-related firing associated with working memory (Zhang et al., 2013) and "strengthened efficacy of dlPFC microcircuit connections, enhancement of mental representations and top-down regulation of behavior" (reviewed in Arnsten et al., 2012 and Arnsten, 2009). These receptors, on the other hand, have inhibitory roles in somatosensory cortices.

The alpha-1 receptor has lower affinity for NE and, when tonically stimulated in the prefrontal cortex (typically under stress), prefrontal top-down control is reduced. In somatosensory cortex, stimulation of alpha- 1 receptors is related to increased neuronal activity. Finally, beta receptors are excitatory and are found in the whole cortex. These receptors are activated under stress but also to support local engagement of highly specialized circuits to process selectively attended information in sensorimotor cortices. Beta-1 receptors have the lowest affinity for NE.

The action of NE on its receptors modulates the response of the neurons to their inputs, which can be seen as "changes in the responses of local circuits" (Phillips et al., 2016). In this way, "NE signaling could be using the differential activities of these receptors to drive many of its well-established network effects" (O'Donnell et al., 2012). NE acts on microcircuits to generate specific effects according to their level of activation (see Section 2.1.3).

LC neurons are the target of many neurochemical systems. In addition to detecting NE (through alpha-2 autoreceptors, which are the most abundant receptors within LC nuclei), they respond to several neurotransmitter and neuromodulators (Table 2). In addition, LC contains receptors for several neuropeptides (for a review see Zitnik, 2016). Since the neuropeptide system is very complex, direct interventions often have antagonistic effects in separate parts of the NE system, and may also lead to severe side effects.

\subsubsection{Tonic and phasic activity of $L C$ neurons}

LC neurons fire in two different modes: tonic and phasic. These modes regulate behavior following an inverted U-shape: a moderate tonic mode allows for maximal phasic mode and optimal performance, while too high or too low tonic mode impairs phasic firing and performance (Fig. 2).

When an animal actively engages in a task, the phasic mode is an instantaneous burst locked to the presentation of a relevant stimulus or to the preparation of a behavioral response. Phasic NE release in the brain increases neural gain in the networks involved in the active

Table 1

Main molecules associated with NE transmission and their cortical effects.

\begin{tabular}{|c|c|c|c|c|}
\hline NE synaptic proteins & G-protein & Affinity to NE & Localization & Effect on cortex \\
\hline$\alpha 2$-receptors & Gi & +++ & $\begin{array}{l}\text { NE axon terminals; LC. } \\
\text { Dendritic spines of PFC pyramidal neurons. }\end{array}$ & $\begin{array}{l}\text { Inhibit NE release. } \\
\text { Increase firing of active populations. }\end{array}$ \\
\hline a1-receptors & $\mathrm{Gq}$ & ++ & Dendritic spines of PFC pyramidal neurons. & Decrease firing. \\
\hline$\beta 1$-receptor & Gs & + & $\begin{array}{l}\text { Dendrites of somato-sensory pyramidal neurons. } \\
\text { Hippocampus }\end{array}$ & $\begin{array}{l}\text { Increase firing. } \\
\text { Increase firing. }\end{array}$ \\
\hline NE transporter (NET) & & & NE axon terminals & Terminate NE and DA transmission \\
\hline
\end{tabular}


Table 2

Membrane receptors present in LC neurons.

\begin{tabular}{lll}
\hline Neurotransmitter/neuromodulator & Receptors in LC & Effect on LC \\
\hline Glutamate & NMDA, AMPA & Excitatory \\
GABA & GABA $_{\mathrm{A}}$ & Inhibitory \\
NE & $\mathrm{Alpha}-2$ & Inhibitory \\
DA & $\mathrm{D}_{1}, \mathrm{D}_{2}$ & Inhibitory \\
Acetylcholine & Nicotinic & Excitatory \\
Serotonin & Serotonin & Inhibitory \\
Orexin & ORX-A & Excitatory \\
Corticotrophin-releasing factor & CRF-1 & Excitatory \\
Glucocorticoids & GR, MR & \\
Opioids & Cannabinoid-1 Mu, Kappa & Inhibitory \\
Substance P & SP & Excitatory \\
Melanin concentrating hormone & MCH-1 & Inhibitory \\
Somatostatin & SS-1 - 3 & Inhibitory \\
Neuropeptide Y & NPY & Inhibitory \\
\hline
\end{tabular}
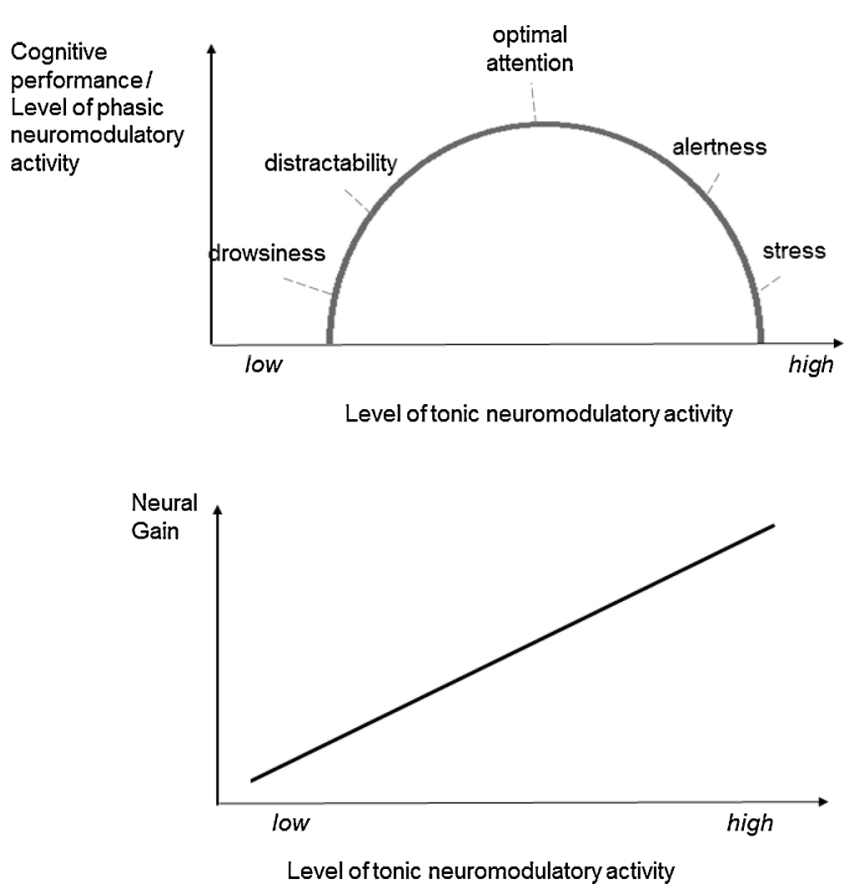

Fig. 2. LC tonic level vs. performance. Level of LC tonic activity determines both phasic level and cognitive performance in an inverted-U shape. This function resembles the Yerkes-Dodson law that describes how performance depends on arousal level (top). Increasing level of LC tonic activity is related to increasing gain in neural circuits (bottom). For a more comprehensive account on LC modes, please refer to (Aston-Jones and Cohen, 2005).

processes at the time of the release. Thus, in a highly active and noisy system, such as the awake brain, NE innervation increases the responsivity of target neurons, selectively potentiating any activity concurrent with LC activation (Berridge and Waterhouse, 2003). At the same time, weak activity is suppressed in a "winner takes all" manner. This enhances the transmission of relevant information and suppresses the transmission of distracting or irrelevant information. The net effect of phasic activation is an increase in the neural signal-to-noise ratio, therefore allowing for more efficient computations: behavioral responses are more efficient, accurate and reliable in the optimal phasic mode (Servan-Schreiber et al., 1990). In short, the phasic mode underlies the increase in neural gain in the brain that allows for efficient cognitive processing ("exploitation") and decision making. Phasic NE suppresses all low and spontaneous activity and leaves intact the strongest evoked responses. A similar signal-to-noise ratio effect of $\mathrm{NE}$ is present in the sensory thalamus (Castro-Alamancos and Calcagnotto, 2001; Hirata and Castro-Alamancos, 2011).
The tonic mode of the LC is the stimulus-independent firing that determines the level of arousal of the organism and triggers exploratory behavior. When measured as multi-unit activity, the tonic firing oscillates at $\sim 2 \mathrm{~Hz}$. However, at the individual level, the frequency is slower than $1 \mathrm{~Hz}$ (Totah et al., 2018). A tonic mode may take a long time to rise and can be maintained until a proper behavioral strategy has been found. During a high tonic mode, the gain of the system is non-selectively high and external stimuli have a lower threshold to reach awareness. This state is sub-optimal if attention needs to be focused, but beneficial when there is a need to flexibly search for strategies. An updated model of the balance between tonic and phasic firing proposes that the transition is determined through modulation of the coupling between neurons in LC and their baseline firing rate (Totah et al., 2018): In an intermediate tonic/optimal phasic mode, large phasic responses and lateral inhibition between LC neurons activate specific brain areas and facilitate exploitative behavior. On the other hand, a high tonic mode is related to a rise in baseline LC activity and low coupling. This mode favors exploratory behavior.

The synaptic mechanisms by which NE increases neural gain in the brain is still an area of active research. A recent model of NE action on target neurons suggests a possible mechanism of interaction between glutamate and NE (GANE) in sensory-motor cortices. The GANE model proposes that glutamate, which signals processing priority in the brain, is associated with highly active neural representations, and that extracellular ("spillover") glutamate stimulates release of $\mathrm{NE}$ under arousal (Mather et al., 2016). NE, in turn, further enhances the release of glutamate in the active synapses with relatively large glutamate concentration via a positive feedback loop that involves NMDA and non-NMDA glutamatergic receptors in NErgic terminals. These active synapses are the sites where relevant information is being processed. At the same time, NE inhibits synapses with weaker activity and low release of glutamate that occur everywhere else in the cortex where nonrelevant information is being processed or spontaneous firing occurs. In this way, NE optimizes the signal-to-noise ratio of glutamatergic transmission in somatosensory cortices (Mather et al., 2016).

\subsubsection{Relation between NE and DA systems and evidence for an LC-DA system}

The LC-NE and ventral tegmental area (VTA)-DA systems share many properties. Both are engaged in response to salient events, are critical for normal PFC function, and have an inverted-U shape doseresponse curve with behavior (see Chandler et al., 2014b for a review of the relation between the two systems). VTA-DA cells also present tonic and phasic modes of firing. LC and VTA are connected through bidirectional projections (Beckstead et al., 1979; Liprando et al., 2004; Mejias-Aponte et al., 2009; for a review of the mutual effects of LC and VTA see El Mansari et al., 2010). Dopaminergic neurons possess alpha-1 and alpha-2 adrenoreceptors (Anden and Grabowska, 1976; Grenhoff et al., 1993), while LC has D2 receptors (Yokoyama et al., 1994). Animal experiments show an inhibitory effect of VTA-DA on LC (Elam et al., 1986; Guiard et al., 2008a, b), probably mediated by alpha-2 adrenoreceptors (Cedarbaum and Aghajanian, 1977). Therefore, the activity in DA and NE systems are intrinsically related, either synergistically or complementarily during and after development (Mokler et al., 2017). In the PFC, NE reuptake proteins catalyze the transport of both DA and NE. It has been shown that DA is taken up in vitro and in vivo by the NE transporter with a potency similar to NE (Rothman et al., 2001; Tanda et al., 1997). In addition, binding studies found that DA and NE have affinity for each other's receptors: NE presents affinity for the D2 family of receptors, and equal affinity as dopamine for D4 receptors, and DA has affinity for alpha2 (Lin et al., 2008; NewmanTancredi et al., 1997; Sánchez-Soto et al., 2016, 2018). NE modulates the release of DA in the PFC (Devoto et al., 2019). Both NE and DA affect the gain of neural circuits, and under stress, the release of both $\mathrm{NE}$ and DA in the hypothalamus and forebrain increases, promoting the characteristic behavior of a stress response. 
On the other hand, the LC and VTA neuromodulatory systems have some specificity in anatomy and function. The NE system projects to the whole cortex, thalamus, and some midbrain structures, while DA mainly projects to premotor and motor areas in primates (Berger et al., 1991), to the medial prefrontal cortex, ACC and entorhinal cortices in rats (Descarries et al., 1987; Lindvall and Bjorklund, 1978) and to the ventral hippocampus in rats (Gasbarri et al., 1994, 1997). At the cellular level, in prefrontal cortices, the activations of alpha-2 and DA-1 receptors have been proposed to exert complementary effects on postsynaptic excitability under normal arousal: alpha-2 receptors increase signal, and DA-1 receptors reduce noise in pyramidal neurons.

Despite the more limited extent of cortical projections of dopaminergic nuclei (VTA), DA receptors are widely spread throughout the cortex (Goldsmith and Joyce, 1994; Lidow et al., 1989; Martres et al., 1985; Richfield et al., 1989). A relatively recent finding is that LC neurons release both NE and DA from their terminals, therefore constituting a source of DA throughout the cortex (Devoto and Flore, 2006; Devoto et al., 2002, 2005; Mejias-Aponte et al., 2009; Smith and Greene, 2012). In fact, during several pharmacological manipulations, Devoto and Flore, 2006 concluded that "in the cerebral cortex, extracellular DA variations better correlate with noradrenergic than with DAergic neuronal activity". Early studies using microdialysis in rats measured similar levels of DA in the whole cortex, and the concentration of DA depended on manipulations not of dopaminergic neurons, but of noradrenergic neurons. Given that DA release by the LC seems to be the norm and not an exception, we can call it the LC-dopaminergic (LC-DA) system. This seems to be the case also in the hippocampus, according to recent studies in rodents. Convergent findings from different labs clearly demonstrate that in the dorsal hippocampus -where pyramidal "place cells" are located-, the main source of DA is the LC (Kempadoo et al., 2016; Takeuchi et al., 2016; Wagatsuma et al., 2018). Furthermore, those studies show that the LC-DA system directly affects the consolidation of memories and the coding of novel context, even at a single-trial level. The mechanisms involve short- and long-term plasticity (Takeuchi et al., 2016).

\subsubsection{Summary}

New theoretical and experimental knowledge on LC-NE system unveils mechanisms by which NE controls the gain of brain circuits in a specific manner to process novel, salient or relevant information, and thus facilitate brain function and cognition.

\subsection{Role of LC-NE system on regulating brain states, cognition, and motivation}

\subsubsection{Arousal and wakefulness}

The role of NE on autonomic arousal and wakefulness has long been known in the field of neurobiology (Berridge et al., 2012; Berridge and Waterhouse, 2003 for reviews). LC is highly, tonically active during awake state, less active during non-REM sleep, and silent during REM sleep. During sleep, an increase in firing of the LC nucleus acts as a wake-promoting signal. Within the awake state, different levels of LC activation are associated to different levels of arousal (Fig. 2). LC contributes to an activated cortex during a vigilant state, with high sensitivity towards incoming information.

\subsubsection{Regulating switches between brain states}

Healthy brain function relies on the ability to efficiently and adaptively switch between different active networks, a process that partly depends on neuromodulation. NE exerts influences on behavior by flexibly engaging neural circuits, especially in the dorso-lateral PFC (Arnsten et al., 2012), to enhance performance on the task at hand. A growing body of literature has implicated NE phasic neuromodulation in the promotion of basic attention functions, such as novelty and saliency detection, sensory processing and orienting response (reviewed by Sara and Bouret 2012). During processing of salient stimuli, LC is recruited through its tight relation with the salience network (including the anterior insula and dorsal anterior cingulate, as well as the amygdala, the ventral striatum, and the brainstem dopaminergic nuclei). Released NE modulates visual and somatosensory cortex activation, boosting activity in whichever functional network is transmitting high priority information (Corbetta et al., 2008). LC activity signals the transition between motor and task-negative networks (Yellin et al., 2015). More broadly, the LC-NE system takes part in the switch between brain modes, a concept captured by the term "network reset" (Guedj et al., 2017; Sara, 2009). Recent work using network approaches to study brain activity indicate that the phasic-tonic balance of LC activity regulates the topography of brain functional networks. Phasic LC activity integrates or coordinates activity in segregated brain regions, that is, regions that would normally operate independently of each other (see Shine, 2019 for a recent review). In this way, phasic NE activity promotes integrative cognitive functions such as executive control and attention.

In addition to task-evoked responses, the "background" brain state or "neuromodulatory environment" (Arnsten et al., 2012) is influenced by the balance between tonic and phasic modes of LC. Indeed, LC "participates in the regulation of pre-attentional/preconscious forms of information processing" (Alsene and Bakshi, 2011) that impact cognitive performance and cognition in general. As mentioned above, performance follows an inverted-U shape with respect to LC tonic firing, where only intermediate levels permit optimal performance and most prominent phasic firing. Low tonic and high tonic LC activity are associated with reduced performance due to a state of drowsiness or anxiousness, respectively. In this regard, cognitive studies on drowsiness or low arousal as well as the literature on stress (an extreme case of hyper-reactivity) add to knowledge of the reduced or impaired cognition at the two extremes of the LC function curve (Howells et al., 2012). Furthermore, work on exploration vs. exploitation balance in complex tasks implicates the LC-NE system (Kane et al., 2017; Sales et al., 2019): while exploitation is characterized by low uncertainty about the optimal way of solving a task and the efficient recruitment of few taskrelated neural circuits, exploration is characterized by the loose recruitment of many neural circuits in order to find the most appropriate strategy, out of many possible of still uncertain utility, for the current goal (Aston-Jones and Cohen, 2005).

In sum, NE acting on its receptors affects the level of neural gain, and modulates the switches between brain networks in accordance with task demands.

\subsubsection{Cognition and motivation}

Work in recent years has focused on studying how LC-NE system facilitates higher-order brain functions. Cell recordings in monkeys and lesion studies have implicated the LC in choice tasks (Aston-Jones et al., 1994; Bouret and Richmond, 2015; Jahn et al., 2018), working memory (WM) (Wang et al., 2007), inhibition, and learning (Bouret and Sara, 2004). In humans, most of the work that points to an involvement of LC in cognition come from pupillometry studies, based on the consistent relationship between LC activity and pupil dilation. Pupil dilation is an indicator of allocation of mental resources or effort (Beatty, 1982; Eckstein et al., 2017; Gilzenrat et al., 2010; Hartmann and Fischer, 2014; Kahneman and Beatty, 1966; Laeng et al., 2012). Pupil dilation correlates with LC spiking (Joshi et al., 2016); therefore, it is relatively well established that pupil dilation reflects LC signaling of novelty and effort in attentional tasks (Murphy et al., 2011). Another proposed neurophysiological correlate of LC postsynaptic effects on cortical activity is the P3 component of event-related potentials (ERP) (Nieuwenhuis et al., 2005; reviewed in Nieuwenhuis, 2011). Although LC activity is not the principal contributor to P3 generation, as it seems to be with the pupil, there is a positive relation between LC and P3 evoked during attentional tasks. More recently, research using neuroimaging techniques have investigated activity in the LC/peri-LC region. Those studies have related LC activation to emotion (Krebs et al., 2018; 
Liddell et al., 2005; Sterpenich et al., 2006), unexpected uncertainty processing (Payzan-LeNestour et al., 2013), focusing and shifting of attention (Von der Gablentz et al., 2015), behavioral flexibility (Clewett et al., 2014), conflict resolution (Köhler et al., 2016), mental effort (Alnæs et al., 2014; Mäki-Marttunen et al., 2020), adaptive flexibility of cognitive control (Mäki-Marttunen et al., 2019) and social behavior (Terbeck et al., 2016). In sum, as posed by Nieuwenhuis, 2011: "it is plausible that noradrenergic system influences prominently the facilitatory influence of the real-life situations, which are characterized by multimodal, crowded sensory environments and a range of potential, often time-consuming response options."

The LC-NE system plays an important role in motivation, particularly in the active engagement of effort. For example, electrophysiological data obtained from monkeys indicated that LC activity mobilizes the sensory and attentional resources needed for processing of a cue and the energy necessary for subsequent action, suggesting that LC-NE activity seems to mediate cognitive effort through moment-tomoment phasic arousal signals as a function of task engagement (Varazzani et al., 2015). The LC-NE system may also be involved in effort-based decision-making. In decision theory, it is assumed that agents compare benefits and costs to estimate a net value of a particular course of action (Glimcher and Fehr, 2013; Pessiglione et al., 2018). Effort-based decision-making refers to a set of mental computations that individuals perform to estimate the amount of work required to obtain an outcome (Culbreth et al., 2018a, 2018b). This framework assumes that agents compare benefits and costs associated with a particular course of action so as to determine the net value of following that course of action. Although the direct involvement of the LC has not been demonstrated, the pupil is sensitive to reward-related processes during decision-making (Van Slooten et al., 2018). Several recent models on decision-making and reinforcement learning speculate that the LC-NE system may act via modulation of anterior cingulate functions (Pessiglione et al., 2018; Silvetti et al., 2018).

A link between LC-NE function and WM capacity (WMC) is put forward in a recent model proposed by Unsworth and Robison, 2017. They propose that the variability in WMC is driven by lapses in attentional control. These lapses reflect the momentary inability of the brain executive networks (fronto-parietal networks - FPN) to suppress the task-negative network (so called default mode network - DMN) and keeping on-task focus (Fig. 3): a weak fronto-parietal system would be less able to suppress distracting stimuli and therefore more lapses in attention would occur, while a stronger FPN system would be more

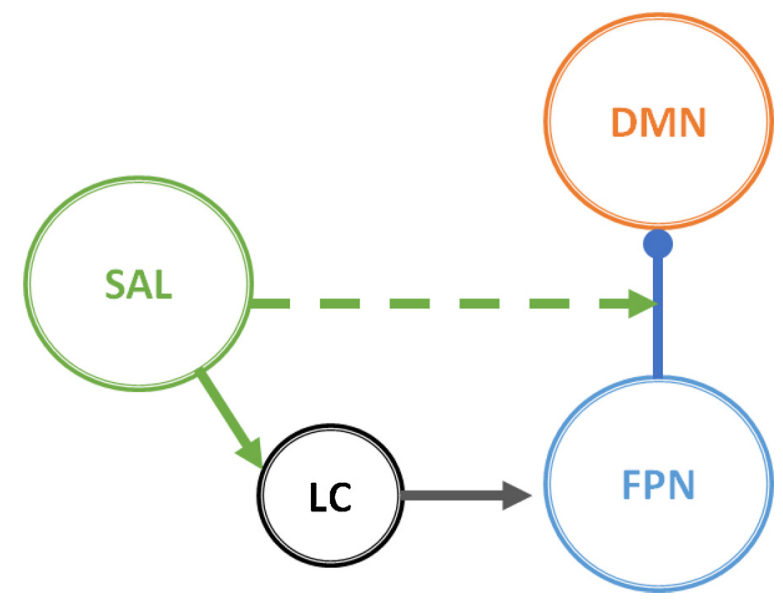

Fig. 3. Model of large-scale networks interaction. The fronto-parietal network (FPN) inhibits the default mode network (DMN) during performance of demanding tasks, driven by the salience network (SAL). An impairment in SALdriven enhancement of FPN activity would lead to a disinhibition of DMN. According to Unsworth and Robison (2017), SAL effect on FPN is mediated by LC recruitment. successful in preventing distracting information from interfering with the task-focused state. Unsworth and Robison propose that the interindividual differences in WMC are explained by differences in the strength of the fronto-parietal system, and that at the same time, this system is directly regulated by the LC-NE system: during a task, moment-to-moment fluctuations in LC activity result in moment-to-moment changes in FPN activity because the NE system has the property of modulating the general processing state of the brain, i.e., the neural gain. The authors further propose that low WMC individuals show a dysregulation of the LC-NE system that takes the brain out of the optimal cognitive state more frequently than in high WMC individuals. An important point made by the authors is that, in addition to the momentto-moment variability in the LC regulation of the FPN, too high or too low activation of the LC would lead to a dysregulation of the FPN following the inverted-U shape curve. Both extremes of LC activation would reduce attentional control and, consequently, performance level. The variability in the LC regulation partly explains the differences between low and high WMC individuals (Aminihajibashi et al., 2019; Tsukahara et al., 2016), and might also underlie the differences in attentional control and performance between healthy individuals and individuals with neurological and psychological brain disorders such as autism, ADHD, or schizophrenia.

As part of the novelty and saliency detection function, LC mediates associative learning of what is salient through long-term plasticity (Markovic et al., 2014). Long-term potentiation (LTP) and long-term depression (LTD) rely on noradrenergic postsynaptic receptors in the hippocampus (Lim et al., 2010). Furthermore, NE plays a role in gating the synthesis of proteins in potentiated circuits and the consequent memory consolidation (Cirelli et al., 1996; Cirelli and Tononi, 2000). This implicates the NE system in long-term changes in synaptic strengths and stabilization of long-term memory. The role of NE in molecular memory consolidation has been proposed earlier (Harley, 2004). Modulatory input from LC is necessary for associative fear conditioning (Johansen et al., 2014). Furthermore, LC activity participates in the different stages of the consolidation of memories: its intervention remains relevant even hours after the memories are created and during sleep following the experience (O'Donnell et al., 2012; Sara, 2015). LC spike timing is phase-locked to hippocampal theta and frontal gamma, and the attentional/network reset function of LC may operate through resetting of theta and gamma frequencies. This may be related to the wiring properties of LC-NE system: LC projections to the frontal cortex are more numerous, fire faster, are more excitable, and contain more glutamate receptors than projections to other regions of the cortex (Chandler et al., 2014a). Increased functional coupling between LC and the frontal cortex was associated with better behavioral performance in the monetary incentive delay task (Clewett et al., 2014), suggesting that the LC-NE system monitors behavioral outcomes and triggers memory processes to modify subsequent behavior.

\subsubsection{Summary}

Different terms have been employed to refer to the role of LC in brain function and behavior: a switch in the trade-off between exploitation and exploration, a brain reset signal, a learning signal or a temporal filter. Frontal areas that are in charge of evaluating the appropriate behavior, for instance anterior cingulate cortex (ACC) and orbitofrontal cortex (OFC), recruit the LC system and induce the appropriate mode of LC function (Aston-Jones and Cohen, 2005). As long as the person is engaged, the NE system covers a wide range of functions from basic perceptual tasks to complex cognition (Chamberlain and Robbins, 2013). Therefore, LC is not involved directly in cognition by performing computations, but it regulates and empowers the computations performed by higher-order areas, making them efficient. The conjunct action of DA and NE achieves the increase in prefrontal signalto-noise ratio (Wang et al., 2007 and Vijayraghavan et al., 2007, reviewed in Robbins and Arnsten, 2009). Importantly, the great flexibility in the dynamic activity and connectivity in the PFC facilitated by 
neuromodulatory systems also makes the circuits highly vulnerable to alterations when this flexibility is lost, contributing to cognitive deficits due to aging or psychosis (Arnsten et al., 2012; Unsworth and Robison, 2017).

\subsection{Developmental aspects and stress}

\subsubsection{The NE system in development}

Developmental changes occur in the noradrenergic and dopaminergic systems (Section 5 in Xing et al., 2016). A recent review exposes the relevant role of the NE system in the development of prefrontal cortices and executive functions (Mokler et al., 2017). The limited research on the developmental trajectory of this system, mostly confined to rodents, suggests that different timing in the development of the different components of the NE system (for instance, levels of the NE transporter (NET) and DA beta-hydroxylase (DBH)) may play a critical role in the differences between reversal learning and reward sensitivity between adolescents and adults, roles that have so far been uniquely attributed to DA. The authors point out holes in our current knowledge and indicate "a need to update work on the anatomy of the noradrenergic systems, transporters and receptors across development with a focus on adolescence. Additionally, it is important to understand how DA and NE interact in various brain regions including the ventral tegmental area and the subregions of the prefrontal cortex across the lifespan to understand how these interactions may influence cognition as well as vulnerability to stress and addiction." For instance, NET density decreases with age in prelimbic and orbital areas from adolescence to adulthood in rats, but not in medial frontal areas. A reduction of NET in frontal areas - where it should not change during adolescence - caused by genetic (Siuta et al., 2010) or other factors, may result in abnormal levels of neurotransmitters and maladaptive compensation mechanisms.

\subsubsection{The NE system in response to stress}

The physiological response to stress depends on the characteristic of the stressor, whether it is acute or chronic. Acute, uncontrollable stress triggers a response mediated by catecholamines and glucocorticoids. Extremely high stress is characterized by strong activation of beta NE receptors (although other neurotransmitters also play a role, such as ACh, histamine and $5 \mathrm{HT}$ ), which leads to high activation and energy consumption from limbic, motor and sensory cortex, and inactivation of the PFC. During stress exposure, cognitive functions that are dependent on the PFC are switched off, such as flexible thinking, rational decision making and WM (for a review, see Hermans et al., 2014). On the other hand, other functions, such as well-rehearsed, rigid behaviors, are performed with greater efficiency after stress. Regions belonging to the salience network, amygdala and hippocampus show enhanced function (attentional vigilance, fear conditioning and consolidation) through acute stress (see Arnsten, 2009 for a review). Glucocorticoids, in an initial phase, potentiate NE and DA effects in different targets, while in a later phase they trigger the reversal of the stress response, the reversal of PFC disconnection, and recovery to a normal state (Hermans et al., 2014).

During exposure to uncontrollable stress, catecholamine levels increase in the brain and initiate intracellular signaling events in prefrontal cortex through adrenoreceptors alpha- 1 and beta- 1 and DA receptor D1 (Deutch et al., 1990; Deutch and Roth, 1990; Finlay and Zigmond, 1997; Roth et al., 1988; Weiss, 1981). The net effect is to switch the PFC off with processes involving increased cAMP signaling. At the same time, sensorimotor systems are switched on through alpha1 receptors and the consolidation of the stressful experience is strengthened through involvement of hippocampus and amygdala. Even though high stress may be beneficial in an acute form, it is detrimental when it is sustained or chronic, and "molecular braking" mechanisms turn down the stress-induced pathways (reviewed in Gamo and Arnsten, 2011). These molecular mechanisms involve DISC1, RGS4 and DGK, which are structural molecules that regulate cAMP concentrations. The DISC1 protein participates in the removal of cAMP when the intracellular levels of this metabolite are high. Therefore, DISC1 is involved in the return from stress-induced cellular changes back to the normal waking state where PFC is functional.

Corticotropin-releasing factor (CRF) is released from the hypothalamus in response to stress and initiates the cascade that leads to cortisol secretion within the hypothalamic-pituitary-adrenal axis. LC neurons receive synaptic contacts with CRF terminals from hypothalamus and other areas, including amygdala. The effect of CRF on LC is an increase in tonic firing, and therefore a reduction in phasic stimulusrelated responses (for a review, see Valentino and Van Bockstaele, 2008; Winklewski et al., 2017; Zitnik, 2016). Opioids produce the opposite effect, inhibiting LC firing and terminating the stress response through $\mu$-opiate receptors located in LC neurons.

Chronic stress causes long-term morphological and functional changes (gene expression, electrophysiological properties) in LC. Studies in rats showed that early adolescence is a period particularly sensitive to the effects of social stress (Bingham et al., 2011). Moreover, social stress in this developmental stage can induce long-lasting morphological and physiological changes in LC. LC increases its tonic activity during exposure to an acute stressor, and increases its local theta synchrony, as well as synchrony with the medial PFC (Zitnik et al., 2016). Exposure to a chronic stressor induces additional changes in LC neurons; receptor and channel trafficking, altered gene expression, and growth of dendrite and axonal sprouts (Borodovitsyna et al., 2018). The morphological changes make the LC more sensitive to stimulation because of more sites of afferent input, and lead to more NE release. Prolonged stress generates structural changes in the PFC and in the catecholaminergic innervations of PFC (Arnsten, 2009). In addition, in early adolescence the response of LC to a stressor is not quenched after removal of the stressors. The theta synchronization with the medial PFC also continues to be present after stressor removal, and is related to impaired coherence in higher frequencies (i.e. gamma, Zitnik et al., 2016). Although it is not clear whether these effects have impact during later developmental stages in healthy animals, they have direct behavioral consequences and may lead to dis-adjustments if interacting with genetic liability.

Interestingly, the dose-response curve of LC to stress-related neurotransmitters changes according to sex, prior stress history (Curtis et al., 1995) and chronic changes in CBF (Butler et al., 1990) or opioid levels.

\subsubsection{Summary}

The LC is a central component in the network of systems that participates in the stress response, interacting with other systems to produce the changes triggered by a stressful event and later to bring the system back to homeostasis. Changes in LC due to stress, in particular in the early windows of susceptibility during development, lead to a higher susceptibility to stressors through increased NE innervation of PFC and impairment of the PFC, which may be expressed, for example, as reduced sustained attention and WM.

\section{The LC-NE system and schizophrenia}

\subsection{Pharmacology of NE system in schizophrenia}

Noradrenergic involvement in SCZ has been proposed for decades, mostly based on pharmacological evidence (Fitzgerald, 2014; Friedman et al., 1999; Fryar-Williams and Strobel, 2015; Lechin and van der Dijs, 2005; Ramos and Arnsten, 2007; Van Kammen and Kelley, 1991; Yamamoto and Hornykiewicz, 2004; Yamamoto et al., 2014). Early evidence suggested that $\mathrm{NE}$ levels are elevated in the cerebro-spinal fluid, brain and serum of patients with SCZ (Bondy et al., 1984; Kemali et al., 1982; reviewed in Van Kammen and Kelley, 1991). Yamamoto et al., 2014 reviewed how different drugs known to modulate SCZ 
symptoms act on DA, NE or both systems. The evidence led them to conclude that the positive symptoms are consistent with a hyperactivity of NE system, while negative symptoms are consistent with a hypoactivity of NE system. In addition, therapeutic effects of certain NEreceptor agonists (such as alpha-2 receptor agonists) have been observed in several studies (for a review see Fitzgerald, 2014), including improvement in positive and cognitive symptoms, as well as in reflexive neurophysiological processes known to be disrupted in SCZ, such as pre-pulse inhibition (PPI) or P50 gating. Studies using antidepressants such as serotonin and NE reuptake inhibitors (SNRIs), suggest that they may have a small but positive effect on cognitive symptoms (Chamberlain and Robbins, 2013; Soczynska et al., 2014; Vernon et al., 2014). Reduced alpha- 2 receptor activity and increased NET activity converge with findings on an animal model of schizophrenia (Siuta et al., 2010). Increased knowledge on pharmacotherapy targeting NE receptors may lead to novel targets in SCZ and other pathologies that present cognitive deficits (Berridge and Spencer, 2016; Ghanemi and Hu, 2015; Maletic et al., 2017).

\subsection{Microcircuit mechanisms}

Two microcircuit hypotheses account for a role of the NE system in PFC dysfunction in SCZ. A recent approach to cellular mechanisms underlying the pathology of SCZ and involving the NE system has been proposed by Phillips et al., 2016. In their detailed work, Philips et al. propose "apical amplification" as the phenomenon linking NE with altered states of consciousness that may underlie the symptoms observed in SCZ. Briefly, the dendrites of L5 pyramidal cells have two compartments, apical and basal, that, thanks to a specific type of cationic channel (hyperpolarization-activated cyclic-nucleotide-gated channel, HCN), can act independently from each other. Furthermore, these compartments receive different types of inputs: while the apical compartment receives inputs that represent the level of arousal, and is influenced by $\mathrm{NE}$, the basal compartment is related to the content of consciousness. A failure in the concerted dynamics of the channels, noradrenergic modulation and synaptic activity in the pyramidal neurons may explain the cognitive symptoms observed in SCZ. The suggested mechanism, involving hyperpolarization-activated (Ih) currents through HCN channels, may be under-regulated by NE in SCZ. A reduced inhibition by alpha- 2 adrenoreceptors of Ih currents leads to increased cAMP signaling, which is known to be increased in SCZ. As the authors propose, this may cause the distractibility and incoherence of thoughts observed in the pathology due to basal dendritic activity (contents) without modulation of apical dendritic activity (context). In this way, this theory relates altered molecular mechanisms with the abnormal context processing observed in SCZ.

Along a similar line, Arnsten et al. (2012) report that the mechanism called "network dynamic connectivity", involving HCN channels, might be under-regulated by noradrenergic and dopaminergic neuromodulators, leading to a failure in optimal PFC function. In thin dendrites of pyramidal cells in the PFC, NE activity through alpha-2 receptors inhibits Ih currents that allow for optimal synaptic conduction between neurons of the same ensembles (i.e., that code for the same stimulus) during focused attention. Similarly, dopaminergic activation of Ih currents through D1 receptors in synapses of neurons of different ensembles increases sensitivity to preferred stimuli in neurons of the PFC. In SCZ, as well as in old age, these mechanisms would be disrupted, with genetic or compensatory alterations that "weaken the regulation of $\mathrm{Ca}^{2+}$-cAMP signaling and/or strengthen the generation of $\mathrm{Ca}^{2+}$-cAMP signaling". Thus, patients would lack the ability to fine-tune circuits to process relevant information, and the authors propose that this is related to the thought disorder observed in the disease.

Krystal et al., 2017 reviewed evidence that circuit properties suffer alterations during the course of SCZ, such as excitatory/inhibitory imbalance and hyperconnectivity. As the authors put it, there is "increased excitability or cortical disinhibition in SCZ, particularly early in the course of the illness", and this brain hyperactivity declines with age and treatment due to compensatory mechanisms. The authors review in detail how these deficits could cause the cognitive impairments observed in SCZ, particularly pointing to deficits in tuning of cortical activity. As suggested by the microcircuit accounts of SCZ, a dysregulated modulation of PFC microcircuit formation by NE and DA, in addition to genetically-labile synaptic function in schizophrenia may lead to the establishment of diffuse circuits lacking specificity. In this way, brain hyperactivity may occur because circuits have not been tightly established.

Further developments in experimental and computational studies may add proof to these promising theories. Such studies will advance our understanding of the influence of NE-mediated effects on positive and cognitive symptoms in SCZ through synaptic mechanisms, i.e. regulation of $\mathrm{Ca}^{2+}$ and Ih channels (Mäki-Marttunen et al., 2017).

\subsection{Modes of LC activation, gain modulation and cognition}

Cognitive deficits precede SCZ onset (Cornblatt et al., 2003, 1999; Erlenmeyer-Kimling et al., 2000; Niendam et al., 2003) and accompany the etiology and course of the illness (Green et al., 2000; Keefe, 2008). $98 \%$ of patients have some degree of cognitive impairment, and cognitive impairments predict poor outcome (Bowie and Harvey, 2005; Green et al., 2004). Cognitive deficits are present in adolescents at risk of SCZ, in patients with first-onset episode, and in their first-degree relatives (Breton et al., 2011; Freedman and Brown, 2011; Kuperberg and Heckers, 2000).

Despite the long-established influence of neuromodulation in SCZ (Yang and Tsai, 2017), very few studies in the field of cognitive neuroscience have been directly designed to test the integrity of the LC-NE system in SCZ. One way to deduce the integrity of LC-NE system in SCZ is by reviewing evidence of markers of LC activity and LC-related functions. As reviewed in Section 2.2.3, light-independent pupil dilation is tightly related to LC function (Larsen and Waters, 2018), and P3 event-related potential constitutes a neurophysiological signature of LC activity in tasks involving LC activation (Murphy et al., 2011). In this section we review evidence providing an indirect but compelling link between LC-NE system and SCZ cognitive function.

First, early on scientists recognized that SCZ patients suffer from an "attentional capacity" deficit, where "attention is a nonspecific but limited resource for cognitive processing. Although its dysfunction will manifest itself in a variety of discrete phenomena, (...) it will be more apparent in tasks that require effortful processing (...)." (Gjerde, 1983). Along this line, when varying task demands in a wide range of tasks, both controls and SCZ patients show capacity overload, but SCZ patients overload at lower levels of demand than controls (although they may still perform normally at the lowest level of demand, i.e., at the easiest level). WM load is one example, where patients show intact performance at low load but impaired performance with increasing load (Deserno et al., 2012; Starc et al., 2017). Also, patients with SCZ are able to track two objects simultaneously but fail to track three or four (a task that is relatively well performed by healthy individuals; Kelemen et al., 2007). The increased effort (that is, requirements of increased gain in perceptual circuits) invested to tackle the increased attentional load (e.g., more objects to track) has been related to increased LC activity (Alnæs et al., 2014; Mäki-Marttunen et al., 2020) and allows for better classification of young and old people using functional connectivity data (Dørum et al., 2016). Studies using pupillometry commonly find increments in pupil dilation during increasingly demanding tasks. These increments are smaller in patients with SCZ as compared to controls (Fish and Granholm, 2008; Granholm et al., 2009, 2016; Granholm and Verney, 2004; Granholm et al., 2007; Minassian et al., 2004; Patterson, 1976; Steinhauer and Hakerem, 1992). Since both pupil dilation and LC activity relate to neural gain (Aston-Jones and Cohen, 2005; Eldar et al., 2013), these results are consistent with SCZ patients having reduced neural gain due to altered 
LC function. Alterations in gain control have been related to SCZ in the dysconnection hypothesis. The dysconnection hypothesis proposes that SCZ arises from a pathological interaction between brain regions due to an aberrant modulation of synaptic efficacy (Friston et al., 2016). The authors propose that "a failure of neuromodulatory gain control translates into a failure to contextualize sensory evidence" (Friston et al., 2016). Both DA and NE are involved in the modulation of neural gain in the PFC. Work directed towards studying neuromodulatory action under load and associated changes in the brain has potential to unravel the mechanisms of cognitive deficits in SCZ.

Second, a number of studies have shown that patients with SCZ have reduced motivation to engage in physical and cognitive activities, particularly when the tasks are experienced as demanding. For example, when offered to perform an easy task for a small reward, or a hard task for a large reward, individuals with SCZ choose the hard task less frequently than healthy controls, and this tendency may scale with the intensity of negative symptoms (Barch et al., 2014; Fervaha et al., 2014; Gold et al., 2015; Horan et al., 2015; Treadway et al., 2015). Patients with SCZ are also biased against cognitive effort. For example, Wolf et al. (2014) showed that patients with SCZ were less willing to exert cognitive effort for monetary rewards in a progressive ratio task, and this effect was stronger for those with more severe negative symptoms. Furthermore, by using a WM-based effort-discounting task Culbreth et al. showed that individuals with SCZ discounted the value of future high reward - high effort trials more strongly than healthy controls (Culbreth et al., 2016). LC is related to reward-related processes during decision-making. Some evidence suggests that NE-related functions are involved in aspects of effort-based decision-making in SCZ. Granholm et al. (2016) tested healthy controls and individuals with SCZ on a digit span task involving three levels of difficulty. The patient group was split in three subgroups based on score on a defeatist beliefs scale. Defeatist performance beliefs are dysfunctional attitudes that one may hold with regards to a particular task, for example "Why bother trying, I always fail," or "It's not worth the effort". The individuals with the highest scores on the defeatist beliefs scale also had the highest level of negative symptoms. During the digit span task, the participants' pupils dilated when holding items in WM, and more strongly when the cognitive load increased. However, the load-dependent pupil dilation was significantly smaller for the group with the most severe defeatist attitudes, suggesting that there is a relationship between reduced effort, negative symptoms, defeatist attitudes, and the activity of the LC-NE system. In this vein, a neuroimaging study of effective connectivity distinguished three subgroups of SCZ patients that mainly differed in the effect of WM load on the strength of frontoparietal and visual-frontal connections (Brodersen et al., 2014). The groups differed in etiological factors such as negative symptoms. Most of the literature on reduced motivation and deficient effort-based decision-making in SCZ focus on dopaminergic functions. However, while there seems to be agreement that DA is a signal of future rewards and initiates action, the role of DA in effort-based decision-making, particularly with regards to the representation of effort cost, and the engagement needed to overcome these, is controversial (Walton and Bouret, 2019). Particularly for the latter function, it has been suggested that the LC-NE system is crucial (Bouret et al., 2012; Varazzani et al., 2015).

Third, a brain-networks perspective of cognitive deficits in SCZ has been proposed based on the triple-network model (Palaniyappan and Liddle, 2012; see Krajcovic et al., 2019 for a recent review). According to this model, the salience network (SAL) regulates the functional balance between the default mode and central executive networks (CEN, including dorsolateral PFC and inferior parietal areas). During cognitive tasks, the SAL would mediate the switch towards CEN activation and concomitant DMN inhibition (Menon and Uddin, 2010; Sridharan et al., 2008). An abnormal function of the SAL would lead to reduced inhibition of the DMN. This reduced inhibition would be related to increased interference by this network and reduced performance. The alteration of this triple system in SCZ has found support in recent work using functional imaging. Supekar et al. (2019) describe larger variability in the cross-network interactions from the SAL in SCZ as compared to controls. Other studies using large samples assessed the connectivity across brain networks in SCZ (Baker et al., 2019; Sheffield et al., 2017). They found an altered function of executive and salience networks in SCZ, supporting a reduced efficiency in the communication between these networks. Given that LC is tightly connected to the function of the SAL (Corbetta et al., 2008), that LC mediates the saliency-driven increase of gain in FPN (Fig. 3), and that phasic NE drives the integration of information across networks (Shine, 2019), further studies assessing the effect of neuromodulation on brain connectivity in SCZ have great potential to provide a more mechanistic view of cognitive deficits in SCZ.

Fourth, several studies have described altered brain oscillatory activity in schizophrenia (Basar and Guntekin, 2008; Koch et al., 2016). A repeated finding is altered gamma power, but also changes in other bands. Interestingly, the precise timing of activity during attention seems to be disrupted across bands, as well as reduced synchrony. As reviewed in Section 2.1.1, LC phasic firing is time-locked to brain oscillations. The proposed role of LC phasic firing in increasing neural gain in a timely manner suggests that it may be at the core of altered brain synchronization and explain at least some cognitive deficits that depend on coordinated brain activity. Given the complexity of the processes underlying EEG activity and cross-frequency coupling, more studies are needed in this direction to draw firmer conclusions.

Fifth, there is some evidence of maladaptive arousal levels in SCZ during cognitive tasks. Decreased fronto-central P300 amplitudes have been found in SCZ patients during oddball and inhibition tasks (Bestelmeyer, 2012; Devrim-Ucok et al., 2006; Ford et al., 1994; Jeon and Polich, 2003; Kiehl et al., 2000; van der Stelt et al., 2004). Howells et al., 2012 proposed that this is indicative of LC hypoactivity. A prediction from a hypotonic mode is that an increased orienting response may favor SCZ patients in tasks where priming helps performance, but would induce increased vulnerability to distraction. Indeed, this seems to be the case in SCZ (Cornblatt et al., 1989; Demeter et al., 2013; Gouzoulis-Mayfrank et al., 2007; Mushquash et al., 2012; Sapir et al., 2007; Spencer et al., 2011). In line with an hypotonic mode, uncertainty-based exploration was reduced in individuals with SZ (Strauss et al., 2011). On the other extreme of the inverted-U curve, high LC activity is involved in the mechanisms of stress and in post-traumatic stress disorder (Atzori et al., 2016). Interestingly, patients with PTSD suffer from auditory hallucinations that are hypothetically similar in phenomenology to those suffered by patients with SCZ (McCarthyJones and Longden, 2015). "Alpha-2 adrenoreceptor antagonist yohimbine produced panic attacks and flashbacks in PTSD subjects. [...] Notably, the flashbacks were specific for each patient such that activating the LC-NE system seemed to trigger the strongly encoded trauma memory but was not the principal mediator of the flashback per se. (Ressler and Nemeroff, 2001)". As mentioned above, patients with schizophrenia suffer hallucinations with greater frequency, and relapses of positive symptoms are more likely to occur after stressful events (Docherty et al., 1978; Szymanski et al., 1983). Alpha-1 low affinity NE receptors are stimulated under stress and their activation impairs PFC function. Atzori et al., 2016 proposed that hyperactivity of the LC-NE system, in combination with disrupted PFC function, may lead to the paranoid and psychotic symptoms of psychiatric diseases, including schizophrenia. High arousal impairs the selectivity of attention (Broadbent, 1971) and therefore the ability to distinguish relevant information from distracting "noise". Gjerde, 1983 reviewed evidence of "hyperarousal as the characteristic arousal pattern of schizophrenics". The author concludes that "the performance of schizophrenics appears to be quite analogous to the performance of essentially normal but highly aroused subjects". Pharmacological studies suggest that there are increased NE levels in cerebro-spinal fluid and serum (Breier et al., 1990; Van Kammen and Kelley, 1991). Paranoid episodes might be 


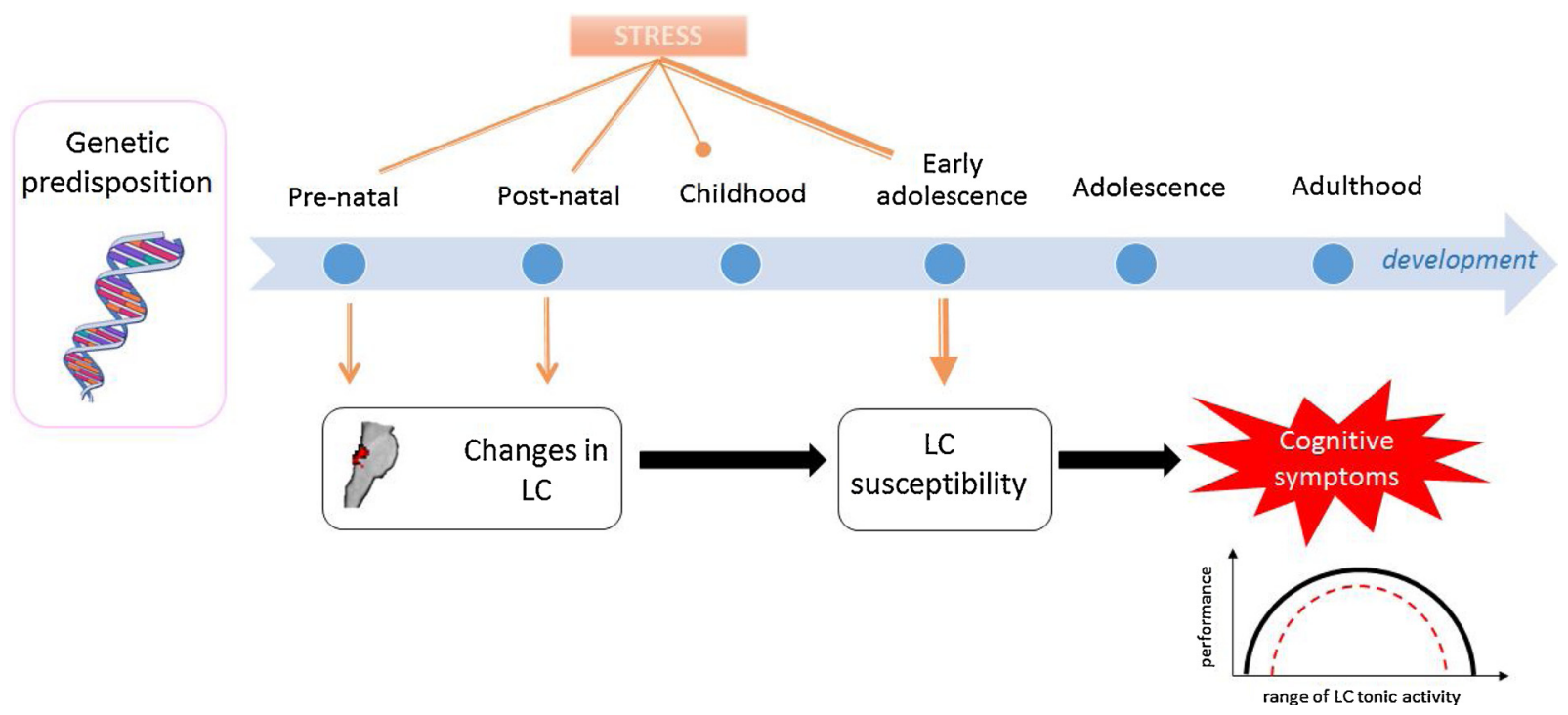

Fig. 4. Effect of stress on the development of LC-NE system and hypothesized interaction with the genetic predisposition to schizophrenia. The interaction between genetics, development and stress appears to lead to the emergence of schizophrenia during adolescence. The LC may suffer functional and morphological changes when exposed to early stress that lead to higher susceptibility during early adolescence, where stress can interact with faulty regulation mechanisms that lead to impaired neuromodulation of the PFC and impaired cognitive function (red dashed curve), i.e. contracted range in the LC function vs. cognitive performance curve of Fig. 2. A contracted range would lead to a reduced range for optimal cognition. During childhood, there is a period of hyposensitivity to stress (blunted arrow).

related to increased NE signaling under stress where PFC function is impaired and the lack of context provided by PFC leads to hallucinations and loss of contact with reality. While evidence for a hypo- and hyper-active NE system in SCZ might seem contradictory, it is important to indicate that the same factors that lead to these contradictions may be the ones that underlie the large heterogeneity of SCZ disease (i.e., genetic profile, patient's history or experience, etc.), in addition to the inherent complexity of the neural machinery. Another factor, time from onset, has been proposed to determine different patient profiles and implicate the NE system (Friedman et al., 1999). As observed by Steinhauer and Hakerem, 1992, "schizophrenia, instead of being a chronically manifest disorder, is episodic in nature, but the vulnerability to the disorder persists". The "episodic nature" of the disease is likely related to metabolic changes: stress leads to high demands of energy and eventually certain metabolites are depleted in the brain and brainstem, leading to changes in NE and other neuromodulators, and behavior (Fryar-Williams and Strobel, 2015).

Given the complexity of brain systems, and the scarcity of studies aimed to directly assess LC-NE effect on cognition in SCZ, it is premature to conclude that the system is altered in SCZ. However, all cognitive functions reviewed to be altered in SCZ (learning, WM, attention and inhibition; Moustafa et al., 2016; Zai et al., 2017) have been robustly linked to LC function, with primary evidence coming from animal models (Logue and Gould, 2014). The circuitry and proposed models suggest that maladaptive changes of the LC-NE system to a dysfunctional cortex may cause a sudden disruption when overexcited by stress, and hypotonic NE activity in the normal awake state may lead to an overexcitable cortex, more variable switching of brain networks, and attentional impairments. In light of the reviewed literature, assessment of the LC-NE system in SCZ appears to be a promising direction.

\subsection{Developmental aspects and stress}

Extensive evidence links history of stress and SCZ (Fitzgerald, 2014; Hammen et al., 1992; Howes and Murray, 2014). Adolescents are exposed to changes in their own body and their social status, which act as stressors that trigger stress responses in their neural and endocrine systems. Other factors accentuate a stressful perception of the environment: "chronic psychosocial stressors like childhood adversity, migration/ethnic minority status and urbanicity have become accepted as increasing the risk of schizophrenia" (Howes et al., 2017). These factors may interplay with genetic susceptibility: Gamo and Arnsten, 2011 point that "many of the molecules that inhibit stress pathways are genetically altered in mental illness, which may explain why these disorders are often precipitated or exacerbated by stress".

Environmental factors can generate stress-evoked pathologies, such as post-traumatic stress disorder (PTSD). They may further interact with physiological factors (such as sexual hormone levels) and genetic factors to produce an abnormal stress response that leads to neuropsychiatric conditions such as SCZ. Certain genetic variations are related to increased stress response or susceptibility. Most of them are related to "molecular brakes", where genetic alterations are implicated in a reduced quenching of the stress response and therefore an exacerbated stress behavior with the associated PFC impairment (Arnsten, 2009). Such variants have been detected in the DISC1, RGS4 and DAKH genes, which, as mentioned in Section 2.3.2, encode molecular brakes that act at some level of the intracellular cascade initiated by activation of adrenoreceptors. Alpha- 2 and possibly beta- 1 pathways are related to DISC1, while alpha-1 is related to RGS4 and DAKH. DISC1 has been related to cortical development (Hikida et al., 2007) and cognitive function (Chubb et al., 2008), and is one of the genes related to SCZ (Cannon et al., 2005). RGS4 is also related to SCZ (Chowdari et al., 2002; Erdely et al., 2006; Mirnics et al., 2001; Morris et al., 2004 reviewed by Arnsten, 2009).

Genetic susceptibility may interact with exposure to stress during developmental periods of high susceptibility. In particular, during early adolescence, prolonged stress is related to structural and functional changes in the rat LC, which lead to impairments in PFC and ultimately cognitive function (Section 2.3). Although the translation of this knowledge to humans is not straightforward, it is plausible that the LC$\mathrm{NE}$ system is not only inherently involved in the development and particularities of the individual expression of SCZ but also in the nature of its evolution and outcome (Fig. 4).

Attempts to explain the underlying deficit of SCZ have suggested that it is not best explained by a focal dysfunction or lesion (a single locus) but by a state of dysconnection, an inability to suppress interference or distracting information and keep context information, or a 
general impairment of neural signal-to-noise ratio (network-level). Further, the developmental nature of SCZ is now recognized (McGlashan and Hoffman, 2000), and neuromodulatory dysfunction may emerge from compensatory processes. The brain constantly adapts to external and internal demands, and this adaptation implicates plastic changes in synapses and changes in the neurochemical environment. The transitions between deep and light sleep and awake states, and between the different arousal states (resting wake, alert focused, mild stress, etc.) are highly coordinated, and protective feedback mechanisms prevent the permanence in extreme states that may damage the neural tissue, for example high stress or too prolonged sustained attention, mechanisms that are expressed with the feeling of fatigue. These adaptations and transitions occur across the whole life in response to external and internal demands. Alterations in the coordination of the different states (for example due to genetically determined factors) may lead to an imbalance in brain homeostasis and structural changes that constrain and determine future processes (Pruessner et al., 2017). It has long been known that NE causes changes in the brain related to stress, and induces WM impairments related to stress (Winklewski et al., 2017). The LC-NE system, as a neuromodulator of the dynamic activity of the whole cortex, could be a key player in the course of SCZ.

\subsection{Relation between $N E$ and other neuromodulators}

The neuromodulatory systems of the brain are highly inter-related and their coordination allows for the different stages of waking vigilance and sleep between which the brain transitions. Therefore, to focus on only one neuromodulatory system, while assuming an intact or at least non-relevant behavior of the others, will give an incomplete view and may even be misleading. Here, we briefly review some proposed hypotheses involving disruption in other neuromodulatory systems in SCZ to provide a more integrated view of the disease.

\subsubsection{ACh}

A recent review has put forward the possibility of a relevant role of the cholinergic pedunculopontine nuclei (PPN) in the pathology of bipolar disorder and SCZ (Garcia-Rill et al., 2015). The coordinated activity of the neurons in these nuclei in gamma rhythm provides "the background gamma activity necessary to support a state capable of reliably assessing the world around us on a continuous basis". The authors review evidence that the activity in the PPN is disrupted in SCZ, potentially leading to disruption of gamma activity and the increased REM sleep drive observed in some of these patients.

The LC projects to the PPN, exerting differential effects on the REMpromoting neurons and wake-promoting neurons of these nuclei (Nieuwenhuys, 1985, reviewed in Szabadi, 2013). Release of NE from LC terminals in the basal forebrain activates cholinergic neurons through alpha- 1 and beta- 1 receptors and inhibits GABAergic neurons through alpha-2 receptors (Berridge and Waterhouse, 2003; Manns et al., 2003; in Schwarz and Luo, 2015). On the other hand, LC neurons express nicotinic Ach receptors, activation of which causes depolarization and an increase in firing rate in rats (Egan and North, 1986). It is well known that patients with SCZ smoke more than the rest of the population. One possibility is that it is a self-therapy phenomenon to counteract the adverse cognitive dysfunctions of the disease. Nicotinic Ach receptors stimulate the release of NE from LC by inducing the release of glutamate (reviewed by dos Santos Coura and Granon, 2012). This offers new avenues for exploring the mechanisms underlying the cognitive dysfunction of SCZ.

\subsection{2. $D A$}

The most investigated hypothesis of schizophrenia is the DAergic hypothesis. The main reason for this is that DA agonists produce psychotic symptoms in healthy subjects resembling those observed in SCZ, while DA antagonists reduce those symptoms. Also, the observed reduction in volume of the ventral tegmental area (VTA) and altered activity in VTA and substantia nigra (SN) in SCZ has added foundation to the hypothesis. However, "genetic findings (...) point to upstream and downstream pathways linked to the dopamine system. A number of the genetic risk factors converge on upstream pathways, particularly those involving glutamatergic systems" (Howes et al., 2017; also see Seeman and Seeman, 2014). Given the large interaction between neuromodulatory systems, it may be rather simplistic to reduce the variety of SCZ symptoms to a sole neuromodulatory system. Abnormal neuromodulation in the brain, by both the DA and NE systems, and probably also by other systems (see, for instance, a serotoninergic account of schizophrenia; Aghajanian and Marek, 2000; Breier, 1995; Eggers, 2013), may offer a more thorough explanation of the disorder, and future studies should address the question of their interaction.

The strong association between DA and SCZ is accommodated in many neurobiological theories of SCZ, such as the dysconnection, context deficit, and aberrant salience hypotheses. The aberrant salience hypothesis proposes that, while DA might not be the primary agent in SCZ, many of the psychotic symptoms are explained by a disruption in the DAergic system. For instance, Kapur, 2003 proposes that this neuromodulatory system mediates the attribution of salience to events and stimuli according to their association to reward or punishment, thus affecting processes such as selective attention, action selection and goal-directed behavior. Strikingly, this is very similar to the affectivebiased hypothesis of noradrenergic action (the BANE model, Markovic et al., 2014). This hypothesis defines affective salience as "the tendency of an item to stand out relative to its neighbors due to an association between its semantic meaning and a history of emotional arousal" (Markovic et al., 2014). Aberrant salience focuses on reward/punishment (i.e., motivational) terms, while affective bias frames it in terms of emotional arousal (i.e. affective). While these two processes may be distinguished and clearly delimited, mechanistically they overlap and are probably highly interconnected in the brain. Further experimental data may allow combining the different neurotransmitter systems into an integral framework so that behavior can be explained in a parsimonious way. For instance, are the processes attributed to one neurotransmitter system mediated by the other or by both? DA (Shizgal, 1997) has been attributed the function of signaling utility while different modes of NE release have separately been attributed the function of mediating utility computations in the ACC and OFC (Aston-Jones and Cohen, 2005).

Work from Bouret and Richmond in monkeys, which was intended to distinguish between the role of DA and NE during an attentional task, suggests that DAergic reward-related release stimulates phasic NE release in LC, but LC also responds independently and under different drives to PFC stimulation, specifically in response to "behavioral energy" (Bouret and Richmond, 2015). In summary, both neuromodulators may have a similar "currency" in the brain (Robbins and Arnsten, 2009), exerting similar effects (changes in gain and networks connectivity), even though their functions diverge in terms of afferent and efferent targets. Further work from animal models with theoreticallydriven experiments will help us understand the specific role of these systems.

The findings on the LC-DA system have motivated studies with second generation antypsychotics. Some recent studies in rats point towards possible roads to pharmacological interventions aimed to selectively enhance noradrenergic-mediated DA transmission in the prefrontal cortex (for example with clozapine or with simultaneous NET and alpha-2 receptor blockers, Devoto et al., 2002; Masana et al., 2011; Vollbrecht, 2010).

\section{Future research directions}

In this review we have gathered recent advances in the knowledge about LC-NE system beyond the traditional views about its limited role on level of wakefulness. We have then reviewed how this system may 
Table 3

Experimental and theoretical progress on LC-NE system and proposed link to SCZ symptoms.

\begin{tabular}{|c|c|}
\hline Advance of knowledge regarding LC-NE system & Relation to schizophrenia \\
\hline $\begin{array}{l}\text { Specificity in LC projections to frontal regions, neuronal constitution and physiology } \\
\text { (Section 2.1.1) }\end{array}$ & $\begin{array}{l}\text { A disruption of the specific action that NE exerts on PFC circuits may underlie } \\
\text { cognitive abnormalities observed in SCZ (Section 3.2). }\end{array}$ \\
\hline LC activity coupled to brain oscillatory activity (Section 2.1.1) & $\begin{array}{l}\text { Signatures of abnormal brain oscillatory activity are related to neurotransmitters and } \\
\text { neuromodulators (Section 3.3) }\end{array}$ \\
\hline NE and DA co-release from LC terminals (Section 2.1.4) & $\begin{array}{l}\text { NE/DA co-release may mediate the action of atypical pharmacological agents } \\
\text { (Section 3.5.2) }\end{array}$ \\
\hline Signatures of LC activity during cognition in humans (Section 2.2.3) & Reduction of pupillometry and P3 signatures in SCZ during attention (Section 3.3) \\
\hline Role of LC in motivation to engage effort (Section 2.2.3) & Reduction of pupillometry signatures with increased effort (Section 3.3) \\
\hline $\begin{array}{l}\text { GANE model of NE-glutamate interplay explains arousal-mediated effect of salient events } \\
\text { (Section 2.1.3) }\end{array}$ & $\begin{array}{l}\text { Altered function of molecules related to synaptic plasticity abnormally interact with } \\
\text { neuromodulation in SCZ (Section 3.2) }\end{array}$ \\
\hline $\begin{array}{l}\text { Network-reset and gain models of the effects of LC-NE system on switching and } \\
\text { enhancement of brain networks' activity (Section 2.2.2) }\end{array}$ & Lower signal-to-noise ratio of cortical circuits in SCZ (Section 3.2) \\
\hline $\begin{array}{l}\text { Models of variability in LC recruitment and regulation of networks interaction explains } \\
\text { traits such as working memory capacity (Section } 2.2 .3 \text { ) }\end{array}$ & Triple-network model and reduced working memory capacity in SCZ (Section 3.3) \\
\hline $\begin{array}{l}\text { Models of how LC-NE system controls the expression of brain states, i.e. stress (Section } \\
\text { 2.3.2) }\end{array}$ & Susceptibility to stress is related to the symptomatology of SCZ (Sections 3.3 and 3.4) \\
\hline
\end{tabular}

Table 4

Methodology to study the integrity of LC-NE system in humans.

\begin{tabular}{|c|c|c|c|}
\hline Method & Relation to LC-NE system & Advantages & Limitations \\
\hline $\begin{array}{l}\text { Pharmacological challenge } \\
\text { (e.g. atomoxetine) }\end{array}$ & $\begin{array}{l}\text { Changes in synaptic concentrations of } \\
\text { NE mimic the effects of elevated/ } \\
\text { reduced NE release by LC. }\end{array}$ & $\begin{array}{l}\text { Allows within-subject comparison of } \\
\text { behavior and physiology (i.e. normal vs. } \\
\text { elevated NE levels). }\end{array}$ & $\begin{array}{l}\text { Pharmacological agents are rarely specific of one } \\
\text { neuromodulatory system and likely interact with other } \\
\text { systems; each individual may have different responsivity; } \\
\text { invasive. }\end{array}$ \\
\hline PET & $\begin{array}{l}\text { NE transporter availability reflects } \\
\text { functionality of LC projections. }\end{array}$ & $\begin{array}{l}\text { Allows for direct molecular } \\
\text { quantification. }\end{array}$ & Very low temporal resolution; invasive. \\
\hline Pupillometry & $\begin{array}{l}\text { Non-reflexive pupil dilation } \\
\text { accompanies LC phasic activation and } \\
\text { tracks changes in tonic LC firing. }\end{array}$ & $\begin{array}{l}\text { Relatively high temporal resolution to } \\
\text { study task-evoked LC activity with low } \\
\text { budget; non-invasive. }\end{array}$ & $\begin{array}{l}\text { Constitutes an indirect measurement of LC activity; other } \\
\text { neuromodulators may contribute to changes in pupil size. }\end{array}$ \\
\hline $\begin{array}{l}\text { Neuromelanin-sensitive } \\
\quad \text { MRI }\end{array}$ & $\begin{array}{l}\text { LC is rich in neuromelanin, a molecule } \\
\text { that is paramagnetic and can be } \\
\text { detected through MRI. }\end{array}$ & $\begin{array}{l}\text { Allows the highest precision in LC } \\
\text { localization in humans in vivo; non- } \\
\text { invasive. }\end{array}$ & $\begin{array}{l}\text { Does not provide information about LC function; intensity } \\
\text { in LC may vary with age. }\end{array}$ \\
\hline fMRI & $\begin{array}{l}\text { Blood oxygen level dependent (BOLD) } \\
\text { changes in LC reflect the level of } \\
\text { neuronal activation in the nuclei. }\end{array}$ & $\begin{array}{l}\text { Allows measuring task-related and } \\
\text { resting state changes of activity with } \\
\text { relatively high spatial resolution; non- } \\
\text { invasive. }\end{array}$ & $\begin{array}{l}\text { Many sources of artifacts affect the quality of the } \\
\text { measurements, i.e. head movement and confounds from } \\
\text { cerebro-spinal fluid vicinity; the small size of LC may also } \\
\text { provide a low signal-to-noise ratio; requires specific } \\
\text { brainstem co-registration steps. }\end{array}$ \\
\hline ERP (P300) & P3 indexes phasic LC response. & $\begin{array}{l}\text { Allows high temporal resolution of a } \\
\text { relatively well characterized component; } \\
\text { non-invasive. }\end{array}$ & LC contributes only in part to the component. \\
\hline
\end{tabular}

have a more central role than previously thought in the symptomatology of SCZ, particularly in relation to cognitive and negative symptoms (for a summary, see Table 3). This is central because cognitive symptoms appear to be the most pervasive and resistant to treatment. Below, we point a series of future directions that we believe can help gain further understanding on SCZ (Table 4).

When it comes to studying the NE system in humans through pharmacology or applying therapies to clinical populations, the antagonistic effects that NE has on its different receptors, side effects and cross-talk with other neuromodulatory systems pose serious limitations. Alternative non-invasive methods such as brain imaging or ocular signatures of LC activity may therefore be particularly useful in research (and possibly in treatment/rehabilitation) on the NE system.

Methods of studying the NE system in humans through pupillometry and neuroimaging have advanced in the past years. For instance, technical developments in brain imaging mean that it is now possible to explore the activity of the brainstem nuclei, and in particular the LC, with MRI to an extent that has not been achieved before in humans. The ability to measure separately the activity in DA and NE nuclei with fMRI may allow us to distinguish between their roles in cognitive processing, and the contribution of these different sources to certain outputs may potentially be assessed and integrated into models. An increasing number of studies focused on the NE system in SCZ, in particular by combining two or more of the available methods
(Table 4), will give us a more complete picture of the disease. Some recent work has been done in this direction (Köhler et al., 2019; Minzenberg et al., 2018). A starting point could be to directly evaluate the triple-networks model (Fig. 3) in SCZ, including the modulatory role of LC.

Another point of focus for future research is the role that neuromodulatory systems have in the diversity of manifestations in SCZ. Each individual brain is shaped according to genetically coded factors, personal experiences, and their interaction. We have reviewed how the LC$\mathrm{NE}$ system plays a role in how personal experiences are wired in the brain (Section 2.2.3), for example in the form of contextual associations (Clewett et al., 2018), decision policies (de Gee et al., 2020), learning mode predisposition (Eldar et al., 2013), or affective biases (Ehlers and Todd, 2017). Work on individual differences in SCZ may build on the knowledge on how LC relates to individual differences in cognitive reserve (Mather and Harley, 2016), WM capacity (Unsworth and Robison, 2015) or sex differences (Herrera et al., 2019). The inclusion of neuromodulatory markers, such as ocular measures (Table 4), will allow inspecting the neurobiological factors contributing to inter-individual differences in SCZ (Diminich and Goff, 2016).

More generally, we propose that future work should focus on studying the NE and other neuromodulatory systems, not in isolation, but in conjunction, in order to unravel the roles that each system has in determining behavior (Storozheva et al., 2015). Future work should 
also scrutinize the specificities and commonalities in the actions of the DA and NE systems. Evidence supports a dysfunction of the DA system in SCZ; however, the DA and NE systems are tightly linked, and their functions partially overlap and partially diverge. DA theories have been largely developed to explain positive symptoms (Stephan et al., 2009). NE has a spatially broader field of action in the brain than DA, and is therefore a suitable candidate for studying cognitive impairments in SCZ. In addition, the conceptualization of the DA system as VTA-DA and LC-DA will add specificity to the assessment of DA function in the brain. We suggest that future studies should assess the complex interplay of the neuromodulatory systems (dos Santos Coura and Granon, 2012; Logue and Gould, 2014) during developmental stages as well as in patients with different symptom types and severity.

\section{Conclusion}

SCZ, rather than having a localized origin, appears to be consistent with a generalized deficit in brain function. Genetic studies have identified multiple gene variants, including genes involved in dopaminergic and glutamatergic neurotransmission and synaptic plasticity. How they can lead to the symptomatology associated to SCZ is still a subject of intense research. Here we propose that SCZ phenomenology, in particular cognitive symptoms, may be explained by an abnormal interaction between genetic susceptibility and stress-initiated LC-NE dysfunction. This in turn, leads to imbalance between tonic and phasic activity modes, dysfunctional regulation of brain network integration and neural gain, and deficits in associated cognitive functions. The LC$\mathrm{NE}$ system is highly centralized and at the same time has the widest outreach in the brain, is highly vulnerable to insults, and is at the core of cognitive deficits observed in many conditions, such as dementia or aging. The interaction between LC-NE system and other neuromodulatory systems may together mediate the environmental effect that precipitates the onset and determines the course of schizophrenia.

\section{Acknowledgements}

This work was funded by grant 231286/F20 (TE) from the Research Council of Norway.

\section{References}

Aghajanian, Marek, 2000. Serotonin model of schizophrenia: emerging role of glutamate mechanisms. Brain Res. Brain Res. Rev. 31 (2-3), 302.

Agster, Mejias-Aponte, Clark, Waterhouse, 2013. Evidence for a regional specificity in the density and distribution of noradrenergic varicosities in rat cortex. J. Comp. Neurol. 521 (10), 2195. https://doi.org/10.1002/cne.23270.

Alnæs, Sneve, Espeseth, Endestad, van de Pavert, Laeng, 2014. Pupil size signals mental effort deployed during multiple object tracking and predicts brain activity in the dorsal attention network and the locus coeruleus. J. Vis. 14, 1.

Alsene, Bakshi, 2011. Pharmacological stimulation of locus coeruleus reveals a new antipsychotic-responsive pathway for deficient sensorimotor gating. Neuropsychopharmacology 36 (8), 1656. https://doi.org/10.1038/npp.2011.47.

Aminihajibashi, Hagen, Foldal, Laeng, Espeseth, 2019. Individual differences in restingstate pupil size: evidence for association between working memory capacity and pupil size variability. Int. J. Psychophysiol. 140, 1. https://doi.org/10.1016/j. ijpsycho.2019.03.007.

Anden, Grabowska, 1976. Pharmacological evidence for a stimulation of dopamine neurons by noradrenaline neurons in the brain. Eur. J. Pharmacol. 39 (2), 275.

Arnsten, 2009. Stress signalling pathways that impair prefrontal cortex structure and function. Nat. Rev. Neurosci. 10 (6), 410. https://doi.org/10.1038/nrn2648.

Arnsten, Wang, Paspalas, 2012. Neuromodulation of thought: flexibilities and vulnerabilities in prefrontal cortical network synapses. Neuron 76 (1), 223. https://doi.org/ 10.1016/j.neuron.2012.08.038.

Aston-Jones, Cohen, 2005. An integrative theory of locus coeruleus-norepinephrine function: adaptive gain and optimal performance. Annu. Rev. Neurosci. 28, 403

Aston-Jones, Waterhouse, 2016. Locus coeruleus: from global projection system to adaptive regulation of behavior. Brain Res. 1645, 75. https://doi.org/10.1016/j. brainres.2016.03.001.

Aston-Jones, Cohen, 2005. Adaptive gain and the role of the locus coeruleus-norepinephrine system in optimal performance. J. Comp. Neurol. 493 (1), 99.

Atzori, Cuevas-Olguin, Esquivel-Rendon, Garcia-Oscos, Salgado-Delgado, Saderi, et al., 2016. Locus ceruleus norepinephrine release: a central regulator of CNS spatiotemporal activation? Front. Synaptic Neurosci. 8, 25. https://doi.org/10.3389/fnsyn.
2016.00025

Baker, Dillon, Patrick, Roffman, Brady, Pizzagalli, et al., 2019. Functional connectomics of affective and psychotic pathology. Proc. Natl. Acad. Sci. 116 (18), 9050.

Barch, Treadway, Schoen, 2014. Effort, anhedonia, and function in schizophrenia: reduced effort allocation predicts amotivation and functional impairment. J. Abnorm. Psychol. 123 (2), 387. https://doi.org/10.1037/a0036299.

Basar, Guntekin, 2008. A review of brain oscillations in cognitive disorders and the role of neurotransmitters. Brain Res. 1235, 172. https://doi.org/10.1016/j.brainres.2008. 06.103.

Beatty, 1982. Task-evoked pupillary responses, processing load, and the structure of processing resources. Psychol. Bull. 91 (2), 276.

Beckstead, Domesick, Nauta, 1979. Efferent connections of the substantia nigra and ventral tegmental area in the rat. Brain Res. 175 (2), 191.

Berger, Gaspar, Verney, 1991. Dopaminergic innervation of the cerebral cortex: unexpected differences between rodents and primates. Trends Neurosci. 14 (1), 21

Berridge, Spencer, 2016. Differential Cognitive Actions of Norepinephrine a2 and a1 Receptor Signaling in the Prefrontal cortex 1641. pp. 189.

Berridge, Waterhouse, 2003. The locus coeruleus-noradrenergic system: modulation of behavioral state and state-dependent cognitive processes. Brain Res. Rev. 42, 33.

Berridge, Schmeichel, Espana, 2012. Noradrenergic modulation of wakefulness/arousal. Sleep Med. Rev. 16 (2), 187. https://doi.org/10.1016/j.smrv.2011.12.003.

Bestelmeyer, 2012. The visual P3a in schizophrenia and bipolar disorder: effects of target and distractor stimuli on the P300. Psychiatry Res. 197 (1-2), 140. https://doi.org/ 10.1016/j.psychres.2011.09.030.

Bingham, McFadden, Zhang, Bhatnagar, Beck, Valentino, 2011. Early adolescence as a critical window during which social stress distinctly alters behavior and brain norepinephrine activity. Neuropsychopharmacology 36 (4), 896.

Bleuler, 1950. Dementia Praecox or the Group of Schizophrenias. International Universities Press, Oxford, England.

Bondy, Ackenheil, Birzle, Elbers, Frohler, 1984. Catecholamines and their receptors in blood: evidence for alterations in schizophrenia. Biol. Psychiatry 19 (10), 1377.

Borodovitsyna, Flamini, Chandler, 2017. Noradrenergic Modulation of Cognition in Health and Disease.

Borodovitsyna, Joshi, Chandler, 2018. Persistent Stress-induced Neuroplastic Changes in the Locus coeruleus/Norepinephrine System.

Bouret, Richmond, 2015. Sensitivity of locus ceruleus neurons to reward value for goaldirected actions. J. Neurosci. 35 (9), 4005. https://doi.org/10.1523/jneurosci.455314.2015 .

Bouret, Ravel, Richmond, 2012. Complementary neural correlates of motivation in dopaminergic and noradrenergic neurons of monkeys. Front. Behav. Neurosci. 6, 40 https://doi.org/10.3389/fnbeh.2012.00040.

Bowie, Harvey, 2005. Cognition in schizophrenia: impairments, determinants, and functional importance. Psychiatr. Clin. North Am. 28 (3), 613. https://doi.org/10 1016/j.psc.2005.05.004.

Braak, Thal, Ghebremedhin, Del Tredici, 2011. Stages of the pathologic process in Alzheimer disease: age categories from 1 to 100 years. J. Neuropathol. Exp. Neurol. 70 (11), 960. https://doi.org/10.1097/NEN.0b013e318232a379.

Breier, 1995. Serotonin, schizophrenia and antipsychotic drug action. Schizophr. Res. 14 (3), 187.

Breier, Wolkowitz, Roy, Potter, Pickar, 1990. Plasma norepinephrine in chronic schizophrenia. Am. J. Psychiatry 147 (11), 1467.

Breton, Plante, Legauffre, Morel, Ades, Gorwood, et al., 2011. The executive control of attention differentiates patients with schizophrenia, their first-degree relatives and healthy controls. Neuropsychologia 49 (2), 203. https://doi.org/10.1016/j. neuropsychologia.2010.11.019.

Broadbent, 1971. Decision and Stress.

Brodersen, Deserno, Schlagenhauf, Lin, Penny, Buhmann, Stephan, 2014. Dissecting psychiatric spectrum disorders by generative embedding. Neuroimage Clin. 4, 98. https://doi.org/10.1016/j.nicl.2013.11.002.

Butler, Weiss, Stout, Nemeroff, 1990. Corticotropin-releasing factor produces fear-enhancing and behavioral activating effects following infusion into the locus coeruleus. J. Neurosci. 10 (1), 176.

Cannon, Hennah, van Erp, Thompson, Lonnqvist, Huttunen, et al., 2005. Association of DISC1/TRAX haplotypes with schizophrenia, reduced prefrontal gray matter, and impaired short- and long-term memory. Arch. Gen. Psychiatry 62 (11), 1205. https:// doi.org/10.1001/archpsyc.62.11.1205.

Cardno, Gottesman, 2000. Twin studies of schizophrenia: from bow-and-arrow concordances to star wars Mx and functional genomics. Am. J. Med. Genet. 97 (1), 12

Carlsson, 1978. Antipsychotic drugs, neurotransmitters, and schizophrenia. Am. J. Psychiatry 135 (2)

Castellanos, Tannock, 2002. Neuroscience of attention-deficit/hyperactivity disorder: the search for endophenotypes. Nat. Rev. Neurosci. 3 (8), 617.

Castro-Alamancos, Calcagnotto, 2001. High-pass filtering of corticothalamic activity by neuromodulators released in the thalamus during arousal: in vitro and in vivo. J. Neurophysiol. 85 (4), 1489. https://doi.org/10.1152/jn.2001.85.4.1489.

Cedarbaum, Aghajanian, 1977. Catecholamine receptors on locus coeruleus neurons: pharmacological characterization. Eur. J. Pharmacol. 44 (4), 375.

Chamberlain, Robbins, 2013. Noradrenergic modulation of cognition: therapeutic implications. J. Psychopharmacol. 27 (8), 694.

Chamberlain, Muller, Blackwell, Robbins, Sahakian, 2006. Noradrenergic modulation of working memory and emotional memory in humans. Psychopharmacology (Berl.) 188 (4), 397. https://doi.org/10.1007/s00213-006-0391-6.

Chandler, Waterhouse, 2012. Evidence for broad versus segregated projections from cholinergic and noradrenergic nuclei to functionally and anatomically discrete subregions of prefrontal cortex. Front. Behav. Neurosci. 6, 20. https://doi.org/10.3389/ fnbeh.2012.00020. 
Chandler, Lamperski, Waterhouse, 2013. Identification and distribution of projections from monoaminergic and cholinergic nuclei to functionally differentiated subregions of prefrontal cortex. Brain Res. 1522, 38. https://doi.org/10.1016/j.brainres.2013. 04.057.

Chandler, Gao, Waterhouse, 2014a. Heterogeneous organization of the locus coeruleus projections to prefrontal and motor cortices. Proc. Natl. Acad. Sci. U. S. A. 111 (18), 6816. https://doi.org/10.1073/pnas.1320827111.

Chandler, Waterhouse, Gao, 2014b. New perspectives on catecholaminergic regulation of executive circuits: evidence for independent modulation of prefrontal functions by midbrain dopaminergic and noradrenergic neurons. Front. Neural Circuits 8, 53. https://doi.org/10.3389/fncir.2014.00053.

Chandler, Jensen, McCall, Pickering, Schwarz, Totah, 2019. Redefining noradrenergic neuromodulation of behavior: impacts of a modular locus coeruleus architecture. J. Neurosci. 39 (42), 8239.

Chowdari, Mirnics, Semwal, Wood, Lawrence, Bhatia, et al., 2002. Association and linkage analyses of RGS4 polymorphisms in schizophrenia. Hum. Mol. Genet. 11 (12), 1373.

Chubb, Bradshaw, Soares, Porteous, Millar, 2008. The DISC locus in psychiatric illness. Mol. Psychiatry 13 (1), 36. https://doi.org/10.1038/sj.mp.4002106.

Cirelli, Tononi, 2000. Gene expression in the brain across the sleep-waking cycle. Brain Res. 885 (2), 303.

Cirelli, Pompeiano, Tononi, 1996. Neuronal gene expression in the waking state: a role for the locus coeruleus. Science 274 (5290), 1211.

Clewett, Schoeke, Mather, 2014. Locus coeruleus neuromodulation of memories encoded during negative or unexpected action outcomes. Neurobiol. Learn. Mem. 111, 65.

Clewett, Huang, Velasco, Lee, Mather, 2018. Locus coeruleus activity strengthens prioritized memories under arousal. J. Neurosci. 38 (6), 1558.

Corbetta, Patel, Shulman, 2008. The reorienting system of the human brain: from environment to theory of mind. Neuron 58 (3), 306.

Cornblatt, Lenzenweger, Erlenmeyer-Kimling, 1989. The continuous performance test, identical pairs version: II. Contrasting attentional profiles in schizophrenic and depressed patients. Psychiatry Res. 29 (1), 65.

Cornblatt, Obuchowski, Roberts, Pollack, Erlenmeyer-Kimling, 1999. Cognitive and behavioral precursors of schizophrenia. Dev. Psychopathol. 11 (3), 487.

Cornblatt, Lencz, Smith, Correll, Auther, Nakayama, 2003. The schizophrenia prodrome revisited: a neurodevelopmental perspective. Schizophr. Bull. 29 (4), 633.

Coull, 1994. Pharmacological manipulations of the alpha 2-noradrenergic system. Effects on cognition. Drugs Aging 5 (2), 116. https://doi.org/10.2165/00002512199405020-00005.

Culbreth, Westbrook, Barch, 2016. Negative symptoms are associated with an increased subjective cost of cognitive effort. J. Abnorm. Psychol. 125 (4), 528. https://doi.org/ $10.1037 /$ abn 0000153 .

Culbreth, Moran, Barch, 2018a. Effort-based decision-making in schizophrenia. Curr. Opin. Behav. Sci. 22, 1. https://doi.org/10.1016/j.cobeha.2017.12.003.

Culbreth, Moran, Barch, 2018b. Effort-cost decision-making in psychosis and depression: could a similar behavioral deficit arise from disparate psychological and neural mechanisms? Psychol. Med. 48 (6), 889. https://doi.org/10.1017/ s0033291717002525.

Curtis, Pavcovich, Grigoriadis, Valentino, 1995. Previous stress alters corticotropin-releasing factor neurotransmission in the locus coeruleus. Neuroscience 65 (2), 541.

Davis, 1991. Dopamine in schizophrenia: a review and reconceptualization. Am. J. Psychiatry 148 (11), 1474.

de Gee, Tsetsos, Schwabe, Urai, McCormick, McGinley, Donner, 2020. Pupil-linked phasic arousal predicts a reduction of choice bias across species and decision domains. Elife 9. https://doi.org/10.7554/eLife.54014.

Demeter, Guthrie, Taylor, Sarter, Lustig, 2013. Increased distractor vulnerability but preserved vigilance in patients with schizophrenia: evidence from a translational Sustained Attention Task. Schizophr. Res. 144 (1-3), 136. https://doi.org/10.1016/j. schres.2013.01.003.

Descarries, Lemay, Doucet, Berger, 1987. Regional and laminar density of the dopamine innervation in adult rat cerebral cortex. Neuroscience 21 (3), 807.

Deserno, Sterzer, Wustenberg, Heinz, Schlagenhauf, 2012. Reduced prefrontal-parietal effective connectivity and working memory deficits in schizophrenia. J. Neurosci. 32 (1), 12. https://doi.org/10.1523/jneurosci.3405-11.2012.

Deutch, Roth, 1990. The determinants of stress-induced activation of the prefrontal cortical dopamine system. Prog. Brain Res. 85, 367.

Deutch, Clark, Roth, 1990. Prefrontal cortical dopamine depletion enhances the respon siveness of mesolimbic dopamine neurons to stress. Brain Res. 521 (1-2), 311.

Devor, Andreassen, Wang, Maki-Marttunen, Smeland, Fan, et al., 2017. Genetic evidence for role of integration of fast and slow neurotransmission in schizophrenia. Mol. Psychiatry 22 (6), 792. https://doi.org/10.1038/mp.2017.33.

Devoto, Flore, 2006. On the origin of cortical dopamine: is it a co-transmitter in noradrenergic neurons? Curr. Neuropharmacol. 4 (2), 115.

Devoto, Flore, Pira, Diana, Gessa, 2002. Co-release of noradrenaline and dopamine in the prefrontal cortex after acute morphine and during morphine withdrawal. Psychopharmacology (Berl.) 160 (2), 220. https://doi.org/10.1007/s00213-0010985-y.

Devoto, Flore, Saba, Fa, Gessa, 2005. Stimulation of the locus coeruleus elicits noradrenaline and dopamine release in the medial prefrontal and parietal cortex. J. Neurochem. 92 (2), 368. https://doi.org/10.1111/j.1471-4159.2004.02866.x.

Devoto, Flore, Saba, Scheggi, Mulas, Gambarana, et al., 2019. Noradrenergic terminals are the primary source of $\alpha 2$-adrenoceptor mediated dopamine release in the medial prefrontal. Cortex 90, 97.

Devrim-Ucok, Keskin-Ergen, Ucok, 2006. Novelty P3 and P3b in first-episode schizophrenia and chronic schizophrenia. Prog. Neuropsychopharmacol. Biol. Psychiatry 30 (8), 1426. https://doi.org/10.1016/j.pnpbp.2006.05.019.
Diminich, Goff, 2016. Is pupillary response a biomarker for effort and defeatist attitudes in patients with schizophrenia? Biol. Psychiatry 80 (8), 574.

Docherty, Van Kammen, Siris, Marder, 1978. Stages of onset of schizophrenic psychosis. Am. J. Psychiatry 135 (4), 420. https://doi.org/10.1176/ajp.135.4.420.

Dørum, Alnæs, Kaufmann, Richard, Lund, Tønnesen, et al., 2016. Age-related differences in brain network activation and co-activation during multiple object tracking. Brain Behav. 6 (11), e00533.

dos Santos Coura, Granon, 2012. Prefrontal neuromodulation by nicotinic receptors for cognitive processes. Psychopharmacology (Berl.) 221 (1), 1. https://doi.org/10. 1007/s00213-011-2596-6.

Eckstein, Guerra-Carrillo, Singley, Bunge, 2017. Beyond eye gaze: What else can eyetracking reveal about cognition and cognitive development? Dev. Cogn. Neurosci. $25,69$.

Egan, North, 1986. Actions of acetylcholine and nicotine on rat locus coeruleus neurons in vitro. Neuroscience 19 (2), 565.

Eggers, 2013. A serotonin hypothesis of schizophrenia. Med. Hypotheses 80 (6), 791. https://doi.org/10.1016/j.mehy.2013.03.013.

Ehlers, Todd, 2017. Genesis and maintenance of attentional biases: the role of the locus coeruleus-noradrenaline system. Neural Plast. 2017, 6817349. https://doi.org/10. $1155 / 2017 / 6817349$.

El Mansari, Guiard, Chernoloz, Ghanbari, Katz, Blier, 2010. Relevance of norepinephrinedopamine interactions in the treatment of major depressive disorder. CNS Neurosci. Ther. 16 (3), e1. https://doi.org/10.1111/j.1755-5949.2010.00146.x.

Elam, Clark, Svensson, 1986. Electrophysiological effects of the enantiomers of 3-PPP on neurons in the locus coeruleus of the rat. Neuropharmacology 25 (9), 1003.

Eldar, Cohen, Niv, 2013. The effects of neural gain on attention and learning. Nat. Neurosci. 16 (8), 1146. https://doi.org/10.1038/nn.3428.

Erdely, Tamminga, Roberts, Vogel, 2006. Regional alterations in RGS4 protein in schizophrenia. Synapse (New York, N.Y.) 59 (8), 472. https://doi.org/10.1002/syn. 20265.

Erlenmeyer-Kimling, Rock, Roberts, Janal, Kestenbaum, Cornblatt, et al., 2000. Attention, memory, and motor skills as childhood predictors of schizophrenia-related psychoses: the New York High-Risk Project. Am. J. Psychiatry 157 (9), 1416. https://doi.org/10. 1176/appi.ajp.157.9.1416.

Fernandes, Christoforou, Giddaluru, Ersland, Djurovic, Mattheisen, et al., 2013. A genetic deconstruction of neurocognitive traits in schizophrenia and bipolar disorder. PLoS One 8 (12), e81052. https://doi.org/10.1371/journal.pone.0081052.

Fervaha, Agid, Foussias, Remington, 2014. Effect of intrinsic motivation on cognitive performance in schizophrenia: a pilot study. Schizophr. Res. 152 (1), 317. https:// doi.org/10.1016/j.schres.2013.11.037.

Finlay, Zigmond, 1997. The effects of stress on central dopaminergic neurons: possible clinical implications. Neurochem. Res. 22 (11), 1387. https://doi.org/10.1023/ a:1022075324164.

Fish, Granholm, 2008. Easier tasks can have higher processing loads: task difficulty and cognitive resource limitations in schizophrenia. J. Abnorm. Psychol. 117 (2), 355. https://doi.org/10.1037/0021-843x.117.2.355.

Fitzgerald, 2014. Is elevated norepinephrine an etiological factor in some cases of schizophrenia? Psychiatry Res. 215 (3), 497.

Folsom, Hawthorne, Lindamer, Gilmer, Bailey, Golshan, et al., 2005. Prevalence and risk factors for homelessness and utilization of mental health services among 10,340 patients with serious mental illness in a large public mental health system. Am. J. Psychiatry 162 (2), 370.

Ford, White, Lim, Pfefferbaum, 1994. Schizophrenics have fewer and smaller P300s: a single-trial analysis. Biol. Psychiatry 35 (2), 96.

Freedman, Brown, 2011. The developmental course of executive functioning in schizophrenia. Int. J. Dev. Neurosci. 29 (3), 237. https://doi.org/10.1016/j.ijdevneu.2010. 11.003.

Friedman, Adler, Davis, 1999. The role of norepinephrine in the pathophysiology of cognitive disorders: potential applications to the treatment of cognitive dysfunction in schizophrenia and Alzheimer's disease. Biol. Psychiatry 46 (9), 1243.

Friston, Brown, Siemerkus, Stephan, 2016. The dysconnection hypothesis (2016). Schizophr. Res. 176 (2-3), 83. https://doi.org/10.1016/j.schres.2016.07.014.

Fryar-Williams, Strobel, 2015. Biomarkers of a five-domain translational substrate for schizophrenia and schizoaffective psychosis. Biomark. Res. 3, 3. https://doi.org/10. 1186/s40364-015-0028-1.

Gamo, Arnsten, 2011. Molecular modulation of prefrontal cortex: rational development of treatments for psychiatric disorders. Behav. Neurosci. 125 (3), 282. https://doi.org/ 10.1037/a0023165.

Garcia-Rill, D’Onofrio, Mahaffey, Bisagno, Urbano, 2015. Pedunculopontine arousal system physiology-Implications for schizophrenia. Sleep Sci. 8 (2), 82. https://doi. org/10.1016/j.slsci.2015.04.003.

Gasbarri, Packard, Campana, Pacitti, 1994. Anterograde and retrograde tracing of projections from the ventral tegmental area to the hippocampal formation in the rat Brain Res. Bull. 33 (4), 445.

Gasbarri, Sulli, Packard, 1997. The dopaminergic mesencephalic projections to the hippocampal formation in the rat. Prog. Neuropsychopharmacol. Biol. Psychiatry 21 (1), 1.

Gesi, Soldani, Giorgi, Santinami, Bonaccorsi, Fornai, 2000. The role of the locus coeruleus in the development of Parkinson's disease. Neurosci. Biobehav. Rev. 24 (6), 655.

Ghanemi, Hu, 2015. Elements toward novel therapeutic targeting of the adrenergic system. Neuropeptides 49, 25. https://doi.org/10.1016/j.npep.2014.11.003.

Gilzenrat, Nieuwenhuis, Jepma, Cohen, 2010. Pupil diameter tracks changes in control state predicted by the adaptive gain theory of locus coeruleus function. Cogn. Affect. Behav. Neurosci. 10 (2), 252. https://doi.org/10.3758/cabn.10.2.252.

Gjerde, 1983. Attentional capacity dysfunction and arousal in schizophrenia. Psychol. Bull. 93 (1), 57. 
Glimcher, Fehr, 2013. Neuroeconomics: Decision Making and the Brain. Academic Press.

Gold, Kool, Botvinick, Hubzin, August, Waltz, 2015. Cognitive effort avoidance and detection in people with schizophrenia. Cogn. Affect. Behav. Neurosci. 15 (1), 145 https://doi.org/10.3758/s13415-014-0308-5.

Goldsmith, Joyce, 1994. Dopamine D2 receptor expression in hippocampus and parahippocampal cortex of rat, cat, and human in relation to tyrosine hydroxylase-immunoreactive fibers. Hippocampus 4 (3), 354. https://doi.org/10.1002/hipo. 450040318.

Gouzoulis-Mayfrank, Balke, Hajsamou, Ruhrmann, Schultze-Lutter, Daumann, Heekeren, 2007. Orienting of attention in unmedicated patients with schizophrenia, prodromal subjects and healthy relatives. Schizophr. Res. 97 (1-3), 35. https://doi.org/10. 1016/j.schres.2007.06.028.

Granholm, Verney, 2004. Pupillary responses and attentional allocation problems on the backward masking task in schizophrenia. Int. J. Psychophysiol. 52 (1), 37. https:// doi.org/10.1016/j.ijpsycho.2003.12.004.

Granholm, Verney, Perivoliotis, Miura, 2007. Effortful cognitive resource allocation and negative symptom severity in chronic schizophrenia. Schizophr. Bull. 33 (3), 831. https://doi.org/10.1093/schbul/sbl040.

Granholm, Fish, Verney, 2009. Pupillometric measures of attentional allocation to target and mask processing on the backward masking task in schizophrenia. Psychophysiology 46 (3), 510.

Granholm, Ruiz, Gallegos-Rodriguez, Holden, Link, 2016. Pupillary responses as a biomarker of diminished effort associated with defeatist attitudes and negative symptoms in schizophrenia. Biol. Psychiatry 80 (8), 581. https://doi.org/10.1016/j. biopsych.2015.08.037.

Green, Kern, Braff, Mintz, 2000. Neurocognitive deficits and functional outcome in schizophrenia: are we measuring the "right stuff"? Schizophr. Bull. 26 (1), 119.

Green, Kern, Heaton, 2004. Longitudinal studies of cognition and functional outcome in schizophrenia: implications for MATRICS. Schizophr. Res. 72 (1), 41.

Grenhoff, Nisell, Ferre, Aston-Jones, Svensson, 1993. Noradrenergic modulation of mid brain dopamine cell firing elicited by stimulation of the locus coeruleus in the rat. Journal of neural transmission. General section 93 (1), 11.

Guedj, Meunier, Meunier, Hadj-Bouziane, 2017. Could LC-NE-Dependent Adjustment of Neural Gain Drive Functional Brain Network Reorganization? Neural Plast. 2017, 4328015. https://doi.org/10.1155/2017/4328015.

Guiard, El Mansari, Blier, 2008a. Cross-talk between dopaminergic and noradrenergic systems in the rat ventral tegmental area, locus ceruleus, and dorsal hippocampus. Mol. Pharmacol. 74 (5), 1463. https://doi.org/10.1124/mol.108.048033.

Guiard, El Mansari, Merali, Blier, 2008b. Functional interactions between dopamine, serotonin and norepinephrine neurons: an in-vivo electrophysiological study in rat with monoaminergic lesions. Int. J. Neuropsychopharmacol. 11 (5), 625. https://doi. org/10.1017/s1461145707008383.

Hammen, Davila, Brown, Ellicott, Gitlin, 1992. Psychiatric history and stress: predictors of severity of unipolar depression. J. Abnorm. Psychol. 101 (1), 45.

Harley, 2004. Norepinephrine and dopamine as learning signals. Neural Plast. 11 (3-4), 191.

Hartmann, Fischer, 2014. Pupillometry: the eyes shed fresh light on the mind. Current biology: CB 24 (7), R281. https://doi.org/10.1016/j.cub.2014.02.028.

Hermans, Henckens, Joels, Fernandez, 2014. Dynamic adaptation of large-scale brain networks in response to acute stressors. Trends Neurosci. 37 (6), 304. https://doi. org/10.1016/j.tins.2014.03.006.

Herrera, Wang, Mather, 2019. The gist and details of sex differences in cognition and the brain: how parallels in sex differences across domains are shaped by the locus coeruleus and catecholamine systems. Prog. Neurobiol. 176, 120.

Hikida, Jaaro-Peled, Seshadri, Oishi, Hookway, Kong, et al., 2007. Dominant-negative DISC1 transgenic mice display schizophrenia-associated phenotypes detected by measures translatable to humans. Proc. Natl. Acad. Sci. U. S. A. 104 (36), 14501 https://doi.org/10.1073/pnas.0704774104.

Hirata, Castro-Alamancos, 2011. Effects of cortical activation on sensory responses in barrel cortex. J. Neurophysiol. 105 (4), 1495. https://doi.org/10.1152/jn.01085. 2010.

Horan, Reddy, Barch, Buchanan, Dunayevich, Gold, et al., 2015. Effort-based decisionmaking paradigms for clinical trials in schizophrenia: part 2-external validity and correlates. Schizophr. Bull. 41 (5), 1055. https://doi.org/10.1093/schbul/sbv090.

Howells, Stein, Russell, 2012. Synergistic tonic and phasic activity of the locus coeruleus norepinephrine (LC-NE) arousal system is required for optimal attentional performance. Metab. Brain Dis. 27 (3), 267. https://doi.org/10.1007/s11011-012-9287-9.

Howes, 2015. Glutamate and dopamine in schizophrenia: an update for the 21 st century. J. Psychopharmacol. 29 (2), 97.

Howes, Murray, 2014. Schizophrenia: an integrated sociodevelopmental-cognitive model. Lancet 383 (9929), 1677.

Howes, McCutcheon, Owen, Murray, 2017. The role of genes, stress, and dopamine in the development of schizophrenia. Biol. Psychiatry 81 (1), 9. https://doi.org/10.1016/j. biopsych.2016.07.014.

Insel, 2010. Rethinking schizophrenia. Nature 468 (7321), 187.

Jaaskelainen, Juola, Hirvonen, McGrath, Saha, Isohanni, et al., 2013. A systematic review and meta-analysis of recovery in schizophrenia. Schizophr. Bull. 39 (6), 1296. https://doi.org/10.1093/schbul/sbs130.

Jacobs, Wiese, van de Ven, Gronenschild, Verhey, Matthews, 2015. Relevance of parahippocampal-locus coeruleus connectivity to memory in early dementia. Neurobiol. Aging 36 (2), 618. https://doi.org/10.1016/j.neurobiolaging.2014.10.041.

Jahn, Gilardeau, Varazzani, Blain, Sallet, Walton, Bouret, 2018. Dual contributions of noradrenaline to behavioural flexibility and motivation. Psychopharmacology 235 (9), 2687.

Jeon, Polich, 2003. Meta-analysis of P300 and schizophrenia: patients, paradigms, and practical implications. Psychophysiology 40 (5), 684.
Jimenez-Rivera, Figueroa, Vazquez-Torres, Velez-Hernandez, Schwarz, VelasquezMartinez, Arencibia-Albite, 2012. Presynaptic inhibition of glutamate transmission by alpha2 receptors in the VTA. Eur. J. Neurosci. 35 (9), 1406. https://doi.org/10. 1111/j.1460-9568.2012.08029.x.

Johansen, Diaz-Mataix, Hamanaka, Ozawa, Ycu, Koivumaa, et al., 2014. Hebbian and neuromodulatory mechanisms interact to trigger associative memory formation. Proc. Natl. Acad. Sci. U. S. A. 111 (51), E5584. https://doi.org/10.1073/pnas. 1421304111.

Joshi, Li, Kalwani, Gold, 2016. Relationships between pupil diameter and neuronal ac tivity in the locus coeruleus, colliculi, and cingulate cortex. Neuron 89 (1), 221.

Kahneman, Beatty, 1966. Pupil diameter and load on memory. Science 154 (3756), 1583.

Kane, Vazey, Wilson, Shenhav, Daw, Aston-Jones, et al., 2017. Increased locus coeruleus tonic activity causes disengagement from a patch-foraging task. Neuroscience 17 (6), 1073. https://doi.org/10.3758/s13415-017-0531-y.

Kapur, 2003. Psychosis as a state of aberrant salience: a framework linking biology, phenomenology, and pharmacology in schizophrenia. Am. J. Psychiatry 160 (1), 13. https://doi.org/10.1176/appi.ajp.160.1.13.

Kebschull, Garcia da Silva, Reid, Peikon, Albeanu, Zador, 2016. High-throughput mapping of single-neuron projections by sequencing of barcoded RNA. Neuron 91 (5), 975. https://doi.org/10.1016/j.neuron.2016.07.036.

Keefe, 2008. Should cognitive impairment be included in the diagnostic criteria for schizophrenia? World Psychiatry 7 (1), 22.

Kelemen, Nagy, Mátyássy, Bitter, Benedek, Vidnyánszky, Kéri, 2007. How well do patients with schizophrenia track multiple moving targets? Neuropsychology 21 (3), 319.

Kemali, Del Vecchio, Maj, 1982. Increased noradrenaline levels in CSF and plasma of schizophrenic patients. Biol. Psychiatry 17 (6), 711.

Kempadoo, Mosharov, Choi, Sulzer, Kandel, 2016. Dopamine release from the locus coeruleus to the dorsal hippocampus promotes spatial learning and memory. Proc. Natl. Acad. Sci. U. S. A. 113 (51), 14835. https://doi.org/10.1073/pnas.1616515114.

Kiehl, Smith, Hare, Liddle, 2000. An event-related potential investigation of response inhibition in schizophrenia and psychopathy. Biol. Psychiatry 48 (3), 210.

Koch, Schmiedt-Fehr, Mathes, 2016. Neuropharmacology of altered brain oscillations in schizophrenia. Int. J. Psychophysiol. 103, 62. https://doi.org/10.1016/j.ijpsycho. 2015.02.014.

Köhler, Bär, Wagner, 2016. Differential involvement of brainstem noradrenergic and midbrain dopaminergic nuclei in cognitive control. Hum. Brain Mapp. 37, 2305.

Köhler, Wagner, Bär, 2019. Activation of brainstem and midbrain nuclei during cognitive control in medicated patients with schizophrenia. Hum. Brain Mapp. 40 (1), 202.

Kraepelin, 1893. Psychiatrie: ein kurzes Lehrbuch für Studirende und Aerzte: Abel.

Krajcovic, Fajnerova, Horacek, Kelemen, Kubik, Svoboda, Stuchlik, 2019. Neural and neuronal discoordination in schizophrenia: from ensembles through networks to symptoms. Acta Physiol. 226 (4), e13282.

Krebs, Park, Bombeke, Boehler, 2018. Modulation of locus coeruleus activity by novel oddball stimuli. Brain Imaging Behav. 12 (2), 577.

Krystal, Anticevic, Yang, Dragoi, Driesen, Wang, Murray, 2017. Impaired tuning of neural ensembles and the pathophysiology of schizophrenia: a translational and computational neuroscience perspective. Biol. Psychiatry 81 (10), 874. https://doi.org/10. 1016/j.biopsych.2017.01.004.

Kuperberg, Heckers, 2000. Schizophrenia and cognitive function. Curr. Opin. Neurobiol. $10(2), 205$

Laeng, Sirois, Gredebäck, 2012. Pupillometry: a window to the preconscious? Perspect. Psychol. Sci. 7 (1), 18.

Larsen, Waters, 2018. Neuromodulatory correlates of pupil dilation. Front. Neural Circuits 12, 21. https://doi.org/10.3389/fncir.2018.00021.

Lechin, van der Dijs, 2005. Noradrenergic hypothesis of schizophrenia. Prog. Neuropsychopharmacol. Biol. Psychiatry 29 (5), 777. https://doi.org/10.1016/j pnpbp.2005.04.003.

Lencz, Knowles, Davies, Guha, Liewald, Starr, et al., 2014. Molecular genetic evidence for overlap between general cognitive ability and risk for schizophrenia: a report from the Cognitive Genomics consorTium (COGENT). Mol. Psychiatry 19 (2), 168. https:// doi.org/10.1038/mp.2013.166.

Liddell, Brown, Kemp, Barton, Das, Peduto, et al., 2005. A direct brainstem-amygdalacortical' alarm' system for subliminal signals of fear. Neuroimage 24 (1), 235. https:// doi.org/10.1016/j.neuroimage.2004.08.016.

Lidow, Goldman-Rakic, Rakic, Innis, 1989. Dopamine D2 receptors in the cerebral cortex: distribution and pharmacological characterization with $[3 \mathrm{H}]$ raclopride. Proc. Natl. Acad. Sci. U. S. A. 86 (16), 6412.

Lieberman, First, 2018. Psychotic disorders. N. Engl. J. Med. 379 (3), 270.

Lim, Tan, Jay, Dawe, 2010. Locus coeruleus stimulation and noradrenergic modulation of hippocampo-prefrontal cortex long-term potentiation. Int. J. Neuropsychopharmacol. 13 (9), 1219. https://doi.org/10.1017/s1461145709991131.

Lin, Quartermain, Dunn, Weinshenker, Stone, 2008. Possible dopaminergic stimulation of locus coeruleus $\alpha 1$-adrenoceptors involved in behavioral activation 62. pp. 5167.

Lindvall, Bjorklund, 1978. Anatomy of the dopaminergic neuron systems in the rat brain. Adv. Biochem. Psychopharmacol. 19, 1.

Liprando, Miner, Blakely, Lewis, Sesack, 2004. Ultrastructural interactions between terminals expressing the norepinephrine transporter and dopamine neurons in the rat and monkey ventral tegmental area. Synapse (New York, N.Y.) 52 (4), 233. https:// doi.org/10.1002/syn.20023.

Lodish, Berk, Zipursky, Matsudaira, Baltimore, Darnell, 2000. Molecular Cell Biology, 4th edition. National Center for Biotechnology Information, Bookshelf.

Logue, Gould, 2014. The neural and genetic basis of executive function: attention, cognitive flexibility, and response inhibition. Pharmacol. Biochem. Behav. 123, 45. https://doi.org/10.1016/j.pbb.2013.08.007.

Mäki-Marttunen, Lines, Edwards, Tveito, Dale, Einevoll, Andreassen, 2017. Pleiotropic 
effects of schizophrenia-associated genetic variants in neuron firing and cardiac pacemaking revealed by computational modeling. Transl. Psychiatry 7 (11), 1.

Mäki-Marttunen, Hagen, Espeseth, 2019. Task context load induces reactive cognitive control: an fMRI study on cortical and brain stem activity. Cogn. Affect. Behav. Neurosci. 1.

Mäki-Marttunen, Hagen, Laeng, Espeseth, 2020. Distinct neural mechanisms meet chal lenges in dynamic visual attention due to either load or object spacing. J. Cogn. Neurosci. 32 (1), 65.

Maletic, Eramo, Gwin, Offord, Duffy, 2017. The role of norepinephrine and its $\alpha$-adrenergic receptors in the pathophysiology and treatment of major depressive disorder and schizophrenia: a systematic review. Front. Psychiatry 8 (42). https://doi.org/10. 3389/fpsyt.2017.00042.

Manns, Lee, Modirrousta, Hou, Jones, 2003. Alpha 2 adrenergic receptors on GABAergic, putative sleep-promoting basal forebrain neurons. Eur. J. Neurosci. 18 (3), 723.

Markovic, Anderson, Todd, 2014. Tuning to the significant: neural and genetic processes underlying affective enhancement of visual perception and memory. Behav. Brain Res. 259, 229.

Martres, Bouthenet, Sales, Sokoloff, Schwartz, 1985. Widespread distribution of brain dopamine receptors evidenced with [125I]iodosulpride, a highly selective ligand. Science 228 (4700), 752.

Marwaha, 2007. Rates and correlates of employment in people with schizophrenia in the UK, France and Germany. Br. J. Psychiatry 191 (1), 30.

Masana, Bortolozzi, Artigas, 2011. Selective enhancement of mesocortical dopaminergic transmission by noradrenergic drugs: therapeutic opportunities in schizophrenia. Int. J. Neuropsychopharmacol. 14 (1), 53. https://doi.org/10.1017/ s1461145710000908.

Mather, Harley, 2016. The Locus coeruleus: essential for maintaining cognitive function and the aging brain. Trends Cogn. Sci. 20 (3), 214.

Mather, Clewett, Sakaki, Harley, 2016. Norepinephrine ignites local hotspots of neuronal excitation: how arousal amplifies selectivity in perception and memory. Behav. Brain Sci. 39, e200. https://doi.org/10.1017/s0140525x15000667.

McCarthy-Jones, Longden, 2015. Auditory verbal hallucinations in schizophrenia and post-traumatic stress disorder: common phenomenology, common cause, common interventions? Front. Psychol. 6, 1071.

McGlashan, Hoffman, 2000. Schizophrenia as a disorder of developmentally reduced synaptic connectivity. Arch. Gen. Psychiatry 57 (7), 637.

McGrath, Saha, Chant, Welham, 2008. Schizophrenia: a concise overview of incidence, prevalence, and mortality. Epidemiol. Rev. 30 (1), 67.

McMillan, White, Franklin, Greenup, Leverenz, Raskind, Szot, 2011. Differential response of the central noradrenergic nervous system to the loss of locus coeruleus neurons in Parkinson's disease and Alzheimer's disease. Brain Res. 1373, 240. https://doi.org/ 10.1016/j.brainres.2010.12.015.

Mejias-Aponte, Drouin, Aston-Jones, 2009. Adrenergic and noradrenergic innervation of the midbrain ventral tegmental area and retrorubral field: prominent inputs from medullary homeostatic centers. J. Neurosci. 29 (11), 3613. https://doi.org/10.1523/ jneurosci.4632-08.2009.

Menon, Uddin, 2010. Saliency, switching, attention and control: a network model of insula function. Brain Struct. Funct. 214 (5-6), 655

Millan, Lejeune, Gobert, 2000. Reciprocal autoreceptor and heteroreceptor control of serotonergic, dopaminergic and noradrenergic transmission in the frontal cortex: relevance to the actions of antidepressant agents. J. Psychopharmacol. (Oxford) 14 (2), 114. https://doi.org/10.1177/026988110001400202.

Minassian, Granholm, Verney, Perry, 2004. Pupillary dilation to simple vs. complex tasks and its relationship to thought disturbance in schizophrenia patients. Int. J. Psychophysiol. 52 (1), 53. https://doi.org/10.1016/j.ijpsycho.2003.12.008.

Minzenberg, Yoon, Soosman, Carter, 2018. Altered brainstem responses to modafinil in schizophrenia: implications for adjunctive treatment of cognition. Transl. Psychiatry 8 (1), 58. https://doi.org/10.1038/s41398-018-0104-z.

Mirnics, Middleton, Stanwood, Lewis, Levitt, 2001. Disease-specific changes in regulator of G-protein signaling 4 (RGS4) expression in schizophrenia. Mol. Psychiatry 6 (3), 293. https://doi.org/10.1038/sj.mp.4000866.

Mokler, Miller, McGaughy, 2017. Evidence for a role of corticopetal, noradrenergic systems in the development of executive function. Neurobiol. Learn. Mem. 143, 94. https://doi.org/10.1016/j.nlm.2017.02.011.

Morris, Rodgers, McGhee, Schwaiger, Scully, Quinn, et al., 2004. Confirming RGS4 as a susceptibility gene for schizophrenia. Am. J. Med. Genet. Part B Neuropsychiatr. Genet. 50 (1). https://doi.org/10.1002/ajmg.b.20109.

Moustafa, Garami, Mahlberg, Golembieski, Keri, Misiak, Frydecka, 2016. Cognitive function in schizophrenia: conflicting findings and future directions. Rev. Neurosci. 27 (4), 435. https://doi.org/10.1515/revneuro-2015-0060.

Murphy, Robertson, Balsters, O'connell, 2011. Pupillometry and P3 index the locus coeruleus-noradrenergic arousal function in humans. Psychophysiology 48 (11), 1532.

Mushquash, Fawcett, Klein, 2012. Inhibition of return and schizophrenia: a meta-analysis. Schizophr. Res. 135 (1-3), 55. https://doi.org/10.1016/j.schres.2011.11.034.

Newman-Tancredi, Audinot-Bouchez, Gobert, Millan, 1997. Noradrenaline and adrenaline are high affinity agonists at dopamine D4 receptors. Eur. J. Pharmacol. 319 (2-3), 379 .

Niendam, Bearden, Rosso, Sanchez, Hadley, Nuechterlein, Cannon, 2003. A prospective study of childhood neurocognitive functioning in schizophrenic patients and their siblings. Am. J. Psychiatry 160 (11), 2060. https://doi.org/10.1176/appi.ajp.160.11 2060 .

Nieuwenhuis, 2011. Investigating the role of the noradrenergic system in human cogni tion. Decision Making, Attention \& Performance. pp. 23.

Nieuwenhuis, Aston-Jones, Cohen, 2005. Decision making, the P3, and the locus coeruleus-norepinephrine system. Psychol. Bull. 131 (4), 510. https://doi.org/10.1037/
0033-2909.131.4.510.

Nieuwenhuys, 1985. Chemoarchitecture of the Brain. Springer-Verlag, Berlin.

Nuechterlein, Subotnik, Ventura, Green, Gretchen-Doorly, Asarnow, 2012. The puzzle of schizophrenia: tracking the core role of cognitive deficits. Dev. Psychopathol. 24 (2), 529. https://doi.org/10.1017/s0954579412000132.

O'Donnell, Zeppenfeld, McConnell, Pena, Nedergaard, 2012. Norepinephrine: a neuromodulator that boosts the function of multiple cell types to optimize CNS performance. Neurochem. Res. 37 (11), 2496. https://doi.org/10.1007/s11064-0120818-x.

Owen, Sawa, Mortensen, 2016. Schizophrenia. Lancet 388, 86.

Owens, Picchioni, Rijsdijk, Stahl, Vassos, Rodger, et al., 2011. Genetic overlap between episodic memory deficits and schizophrenia: results from the Maudsley Twin Study. Psychol. Med. 41 (3), 521. https://doi.org/10.1017/S0033291710000942.

Palaniyappan, Liddle, 2012. Does the salience network play a cardinal role in psychosis? An emerging hypothesis of insular dysfunction. J. Psychiatry Neurosci.

Patterson, 1976. Skin conductance responding/nonresponding and pupiliometrics in chronic schizophrenia. A confirmation of Gruzelier and Venables. J. Nerv. Ment. Dis. 163 (3), 200.

Payzan-LeNestour, Dunne, Bossaerts, O'Doherty, 2013. The neural representation of unexpected uncertainty during value-based decision making. Neuron 79, 191.

Pessiglione, Vinckier, Bouret, Daunizeau, Le Bouc, 2018. Why not try harder? Computational approach to motivation deficits in neuro-psychiatric diseases. Brain 141 (3), 629. https://doi.org/10.1093/brain/awx278.

Phillips, Larkum, Harley, Silverstein, 2016. The effects of arousal on apical amplification and conscious state. Neurosci. Conscious. 2016 (1), niw015. https://doi.org/10. 1093/nc/niw015.

Pruessner, Cullen, Aas, Walker, 2017. The neural diathesis-stress model of schizophrenia revisited: an update on recent findings considering illness stage and neurobiological and methodological complexities. Neurosci. Biobehav. Rev. 73, 191.

Ramos, Arnsten, 2007. Adrenergic pharmacology and cognition: focus on the prefrontal cortex. Pharmacol. Ther. 113 (3), 523. https://doi.org/10.1016/j.pharmthera.2006. 11.006.

Ressler, Nemeroff, 2001. Role of norepinephrine in the pathophysiology of neuropsychiatric disorders. CNS Spectr. 6 (8), 663.

Richards, Pardinas, Frizzati, Tansey, Lynham, Holmans, et al., 2019. The Relationship Between Polygenic Risk Scores and Cognition in Schizophrenia. Schizophr. Bull. https://doi.org/10.1093/schbul/sbz061.

Richfield, Young, Penney, 1989. Comparative distributions of dopamine D-1 and D-2 receptors in the cerebral cortex of rats, cats, and monkeys. J. Comp. Neurol. 286 (4), 409. https://doi.org/10.1002/cne.902860402.

Robbins, Arnsten, 2009. The neuropsychopharmacology of fronto-executive function: monoaminergic modulation. Annu. Rev. Neurosci. 32, 267. https://doi.org/10.1146/ annurev.neuro.051508.135535.

Robertson, Plummer, de Marchena, Jensen, 2013. Developmental origins of central norepinephrine neuron diversity. Nat. Neurosci. 16 (8), 1016. https://doi.org/10.1038/ nn.3458.

Rossler, Salize, van Os, Riecher-Rossler, 2005. Size of burden of schizophrenia and psychotic disorders. Eur. Neuropsychopharmacol. 15 (4), 399. https://doi.org/10.1016/ j.euroneuro.2005.04.009.

Roth, Tam, Ida, Yang, Deutch, 1988. Stress and the mesocorticolimbic dopamine systems. Ann. N. Y. Acad. Sci. 537, 138.

Rothman, Baumann, Dersch, Romero, Rice, Carroll, Partilla, 2001. Amphetamine-type central nervous system stimulants release norepinephrine more potently than they release dopamine and serotonin. Synapse (New York, N.Y.) 39 (1), 32. https://doi. org/10.1002/1098-2396(20010101)39:1 < 32::Aid-syn5 > 3.0.Co;2-3.

Sales, Friston, Jones, Pickering, Moran, 2019. Locus Coeruleus tracking of prediction errors optimises cognitive flexibility: an Active Inference model. PLoS Comput. Biol. 15 (1), e1006267. https://doi.org/10.1371/journal.pcbi.1006267.

Sánchez-Soto, Bonifazi, Cai, Ellenberger, Newman, Ferré, Yano, 2016. Evidence for noncanonical neurotransmitter activation: norepinephrine as a dopamine D2-like receptor agonist. Mol. Pharmacol. 89 (4), 457.

Sánchez-Soto, Casadó-Anguera, Yano, Bender, Cai, Moreno, et al., 2018. $\alpha 2 \mathrm{~A}$ - and $\alpha 2 \mathrm{C}$ adrenoceptors as potential targets for dopamine and dopamine receptor ligands. Mol. Neurobiol. 55 (11), 8438. https://doi.org/10.1007/s12035-018-1004-1.

Sapir, Dobrusin, Ben-Bashat, Henik, 2007. Neuroleptics reverse attentional effects in schizophrenia patients. Neuropsychologia 45 (14), 3263. https://doi.org/10.1016/j. neuropsychologia.2007.06.007.

Sara, 2009. The locus coeruleus and noradrenergic modulation of cognition. Nat. Rev. Neurosci. 10 (3), 211. https://doi.org/10.1038/nrn2573.

Sara, 2015. Locus Coeruleus in time with the making of memories. Curr. Opin. Neurobiol. 35, 87. https://doi.org/10.1016/j.conb.2015.07.004.

Scheibner, Trendelenburg, Hein, Starke, 2001. Alpha2-adrenoceptors modulating neuronal serotonin release: a study in alpha2-adrenoceptor subtype-deficient mice. Br. J. Pharmacol. 132 (4), 925. https://doi.org/10.1038/sj.bjp.0703882.

Schwarz, Luo, 2015. Organization of the locus coeruleus-norepinephrine system. Curr Biol.: CB 25 (21), R1051. https://doi.org/10.1016/j.cub.2015.09.039.

Seeman, Seeman, 2014. Is schizophrenia a dopamine supersensitivity psychotic reaction? Prog. Neuropsychopharmacol. Biol. Psychiatry 48, 155. https://doi.org/10.1016/j. pnpbp. 2013.10.003.

Servan-Schreiber, Printz, Cohen, 1990. A network model of catecholamine effects: gain, signal-to-noise ratio, and behavior. Science 249 (4971), 892.

Sheffield, Kandala, Tamminga, Pearlson, Keshavan, Sweeney, et al., 2017 Transdiagnostic associations between functional brain network integrity and cognition. JAMA Psychiatry 74 (6), 605.

Shields, Wang, Winder, 2009. alpha2A-adrenergic receptors heterosynaptically regulate glutamatergic transmission in the bed nucleus of the stria terminalis. Neuroscience 
163 (1), 339. https://doi.org/10.1016/j.neuroscience.2009.06.022.

Shine, 2019. Neuromodulatory influences on integration and segregation in the brain. Trends Cogn. Sci.

Shizgal, 1997. Neural basis of utility estimation. Curr. Opin. Neurobiol. 7 (2), 198.

Silvetti, Vassena, Abrahamse, Verguts, 2018. Dorsal anterior cingulate-brainstem ensemble as a reinforcement meta-learner. PLoS Comput. Biol. 14 (8), e1006370. https://doi.org/10.1371/journal.pcbi.1006370.

Siuta, Robertson, Kocalis, Saunders, Gresch, Khatri, et al., 2010. Dysregulation of the norepinephrine transporter sustains cortical hypodopaminergia and schizophrenialike behaviors in neuronal rictor null mice. PLoS Biol. 8 (6), e1000393. https://doi. org/10.1371/journal.pbio.1000393.

Smeland, Andreassen, 2018. How can genetics help understand the relationship between cognitive dysfunction and schizophrenia? Scand. J. Psychol. 59 (1), 26. https://doi. org/10.1111/sjop.12407.

Smeland, Frei, Kauppi, Hill, Li, Wang, et al., 2017. Identification of genetic loci jointly influencing schizophrenia risk and the cognitive traits of verbal-numerical reasoning, reaction time, and general cognitive function. JAMA Psychiatry 74 (10), 1065. https://doi.org/10.1001/jamapsychiatry.2017.1986.

Smeland, Bahrami, Frei, Shadrin, O'Connell, Savage, et al., 2019. Genome-wide analysis reveals extensive genetic overlap between schizophrenia, bipolar disorder, and intelligence. Mol. Psychiatry. https://doi.org/10.1038/s41380-018-0332-x.

Smith, Greene, 2012. CNS dopamine transmission mediated by noradrenergic innervation. J. Neurosci. 32 (18), 6072. https://doi. org/10.1523/jneurosci.6486-11.2012.

Soczynska, Ravindran, Styra, Mc Intyre, Cyriac, Manierka, Kennedy, 2014. The effect of bupropion XL and escitalopram on memory and functional outcomes in adults with major depressive disorder: results from a randomized controlled trial. Psychiatry Res. 220 (1-2), 245. https://doi.org/10.1016/j.psychres.2014.06.053.

Spencer, Nestor, Valdman, Niznikiewicz, Shenton, McCarley, 2011. Enhanced facilitation of spatial attention in schizophrenia. Neuropsychology 25 (1), 76. https://doi.org/10. 1037/a0020779.

Sridharan, Levitin, Menon, 2008. A critical role for the right fronto-insular cortex in switching between central-executive and default-mode networks. Proc. Natl. Acad. Sci. 105 (34), 12569.

Starc, Murray, Santamauro, Savic, Diehl, Cho, et al., 2017. Schizophrenia is associated with a pattern of spatial working memory deficits consistent with cortical disinhibition. Schizophr. Res. 181, 107. https://doi.org/10.1016/j.schres.2016.10.011.

Stein, Wise, 1971. Possible etiology of schizophrenia: progressive damage to the noradrenergic reward system by 6-hydroxydopamine. Science 171 (3975), 1032.

Steinhauer, Hakerem, 1992. The pupillary response in cognitive psychophysiology and schizophrenia. Ann. N. Y. Acad. Sci. 658, 182.

Stephan, Friston, Frith, 2009. Dysconnection in schizophrenia: from abnormal synaptic plasticity to failures of self-monitoring. Schizophr. Bull. 35 (3), 509. https://doi.org/ 10.1093/schbul/sbn176.

Sterpenich, D’Argembeau, Desseilles, Balteau, Albouy, Vandewalle, et al., 2006. The locus ceruleus is involved in the successful retrieval of emotional memories in humans. J. Neurosci. 26, 7416.

Storozheva, Kirenskaya, Proshin, 2015. The neuromediator mechanisms of the cognitive deficit in schizophrenia. Neurochem. J. 9 (3), 186.

Strauss, Frank, Waltz, Kasanova, Herbener, Gold, 2011. Deficits in positive reinforcement learning and uncertainty-driven exploration are associated with distinct aspects of negative symptoms in schizophrenia. Biol. Psychiatry 69 (5), 424.

Sullivan, Kendler, Neale, 2003. Schizophrenia as a complex trait: evidence from a metaanalysis of twin studies. Arch. Gen. Psychiatry 60 (12), 1187.

Supekar, Cai, Krishnadas, Palaniyappan, Menon, 2019. Dysregulated brain dynamics in a triple-network saliency model of schizophrenia and its relation to psychosis. Biol. Psychiatry 85 (1), 60.

Szabadi, 2013. Functional neuroanatomy of the central noradrenergic system. J. Psychopharmacol. (Oxford, England) 27 (8), 659. https://doi.org/10.1177/ 0269881113490326.

Szot, Miguelez, White, Franklin, Sikkema, Wilkinson, et al., 2010. A comprehensive analysis of the effect of DSP4 on the locus coeruleus noradrenergic system in the rat. Neuroscience 166 (1), 279. https://doi.org/10.1016/j.neuroscience.2009.12.027.

Szymanski, Simon, Gutterman, 1983. Recovery from schizophrenic psychosis. Am. J. Psychiatry 140 (3), 335. https://doi.org/10.1176/ajp.140.3.335.

Takeuchi, Duszkiewicz, Sonneborn, Spooner, Yamasaki, Watanabe, et al., 2016. Locus coeruleus and dopaminergic consolidation of everyday memory. Nature 537 (7620), 357. https://doi.org/10.1038/nature19325.

Tanda, Pontieri, Frau, Di Chiara, 1997. Contribution of blockade of the noradrenaline carrier to the increase of extracellular dopamine in the rat prefrontal cortex by amphetamine and cocaine. Eur. J. Neurosci. 9 (10), 2077.

Terbeck, Savulescu, Chesterman, Cowen, 2016. Noradrenaline effects on social behaviour, intergroup relations, and moral decisions. Neurosci. Biobehav. Rev. 66, 54. https://doi.org/10.1016/j.neubiorev.2016.03.031.

Totah, Neves, Panzeri, Logothetis, Eschenko, 2018. The locus coeruleus is a complex and differentiated neuromodulatory system. Neuron 99 (5), 1055. https://doi.org/10. 1016/j.neuron.2018.07.037.

Toulopoulou, Picchioni, Rijsdijk, Hua-Hall, Ettinger, Sham, Murray, 2007. Substantial genetic overlap between neurocognition and schizophrenia: genetic modeling in twin samples. Arch. Gen. Psychiatry 64 (12), 1348. https://doi.org/10.1001/archpsyc.64. 12.1348.

Treadway, Peterman, Zald, Park, 2015. Impaired effort allocation in patients with schizophrenia. Schizophr. Res. 161 (2-3), 382. https://doi.org/10.1016/j.schres.2014.11 024.

Tsukahara, Harrison, Engle, 2016. The relationship between baseline pupil size and intelligence. Cogn. Psychol. 91, 109. https://doi.org/10.1016/j.cogpsych.2016.10.001.
Uematsu, Tan, Ycu, Cuevas, Koivumaa, Junyent, et al., 2017. Modular organization of the brainstem noradrenaline system coordinates opposing learning states. Nat. Neurosci. 20, 1602. https://doi.org/10.1038/nn.4642.

Unsworth, Robison, 2015. Individual differences in the allocation of attention to items in working memory: evidence from pupillometry. Psychon. Bull. Rev. 22 (3), 757.

Unsworth, Robison, 2017. The importance of arousal for variation in working memory capacity and attention control: a latent variable pupillometry study. J. Exp. Psychol. Learn. Mem. Cogn.

Valentino, Van Bockstaele, 2008. Convergent regulation of locus coeruleus activity as an adaptive response to stress. Eur. J. Pharmacol. 583 (2-3), 194. https://doi.org/10. 1016/j.ejphar.2007.11.062.

van der Stelt, Frye, Lieberman, Belger, 2004. Impaired P3 generation reflects high-level and progressive neurocognitive dysfunction in schizophrenia. Arch. Gen. Psychiatry 61 (3), 237. https://doi.org/10.1001/archpsyc.61.3.237.

Van Kammen, Kelley, 1991. Dopamine and norepinephrine activity in schizophrenia: an intergrative perspective. Schizophr. Res. 4 (2), 173.

Van Slooten, Jahfari, Knapen, Theeuwes, 2018. How pupil responses track value-based decision-making during and after reinforcement learning. PLoS Comput. Biol. 14 (11), e1006632. https://doi.org/10.1371/journal.pcbi.1006632.

Varazzani, San-Galli, Gilardeau, Bouret, 2015. Noradrenaline and dopamine neurons in the reward/effort trade-off: a direct electrophysiological comparison in behaving monkeys. J. Neurosci. 35 (20), 7866

Vernon, Grudnikoff, Seidman, Frazier, Vemulapalli, Pareek, et al., 2014. Antidepressants for cognitive impairment in schizophrenia-a systematic review and meta-analysis. Schizophr. Res. 159 (2-3), 385. https://doi.org/10.1016/j.schres.2014.08.015.

Vijayraghavan, Wang, Birnbaum, Williams, Arnsten, 2007. Inverted-U dopamine D1 receptor actions on prefrontal neurons engaged in working memory. Nat. Neurosci. 10 (3), 376. https://doi.org/10.1038/nn1846.

Vollbrecht, 2010. Mechanisms for the interaction of dopamine and norepinephrine in the prefrontal cortex: implications for the treatment of cognitive symptoms of schizophrenia. Vanderbilt Reviews Neuroscience 2 Retrieved from Vanderbilt Reviews Neuroscience website:

Von der Gablentz, Tempelmann, Münte, Heldmann, 2015. Performance monitoring and behavioral adaptation during task switching: an fMRI study. Neuroscience 285, 227.

Wagatsuma, Okuyama, Sun, Smith, Abe, Tonegawa, 2018. Locus coeruleus input to hippocampal CA3 drives single-trial learning of a novel context. Proc. Natl. Acad. Sci. U. S. A. 115 (2), E310. https://doi.org/10.1073/pnas.1714082115.

Walton, Bouret, 2019. What Is the Relationship between Dopamine and Effort? Trends Neurosci. 42 (2), 79. https://doi.org/10.1016/j.tins.2018.10.001.

Wang, Ramos, Paspalas, Shu, Simen, Duque, et al., 2007. Alpha2A-adrenoceptors strengthen working memory networks by inhibiting cAMP-HCN channel signaling in prefrontal cortex. Cell 129 (2), 397. https://doi.org/10.1016/j.cell.2007.03.015.

Weinshenker, 2008. Functional consequences of locus coeruleus degeneration in Alzheimer's disease. Curr. Alzheimer Res. 5 (3), 342.

Weinshenker, Holmes, 2016. Regulation of neurological and neuropsychiatric phenotypes by locus coeruleus-derived galanin. Brain Res. 1641 (Pt B), 320. https://doi.org/10. 1016/j.brainres.2015.11.025.

Weiss, 1981. Behavioral depression produced by an uncontrollable stressor: relationship to norepinephrine, dopamine, and serotonin levels in various regions of rat brain. Brain Res. Rev. 3 (2).

Winklewski, Radkowski, Wszedybyl-Winklewska, Demkow, 2017. Stress response, brain noradrenergic system and cognition. Adv. Exp. Med. Biol. 980, 67. https://doi.org/ 10.1007/5584_2016_204.

Wolf, Satterthwaite, Kantrowitz, Katchmar, Vandekar, Elliott, Ruparel, 2014. Amotivation in schizophrenia: integrated assessment with behavioral, clinical, and imaging measures. Schizophr. Bull. 40 (6), 1328. https://doi.org/10.1093/schbul/ sbu026.

Xing, Li, Gao, 2016. Norepinephrine versus dopamine and their interaction in modulating synaptic function in the prefrontal cortex. Brain Res. 1641 (Pt B), 217. https://doi. org/10.1016/j.brainres.2016.01.005.

Yamamoto, Hornykiewicz, 2004. Proposal for a noradrenaline hypothesis of schizophrenia. Prog. Neuropsychopharmacol. Biol. Psychiatry 28 (5), 913. https://doi.org/ 10.1016/j.pnpbp.2004.05.033.

Yamamoto, Shinba, Yoshii, 2014. Psychiatric symptoms of noradrenergic dysfunction: a pathophysiological view. Psychiatry Clin. Neurosci. 68 (1), 1.

Yang, Tsai, 2017. New targets for schizophrenia treatment beyond the dopamine hypothesis. Int. J. Mol. Sci. 18 (8). https://doi.org/10.3390/ijms18081689.

Yellin, Berkovich-Ohana, Malach, 2015. Coupling between pupil fluctuations and restingstate fMRI uncovers a slow build-up of antagonistic responses in the human cortex. Neuroimage 106, 414. https://doi.org/10.1016/j.neuroimage.2014.11.034.

Yokoyama, Okamura, Nakajima, Taguchi, Ibata, 1994. Autoradiographic distribution of [3H]YM-09151-2, a high-affinity and selective antagonist ligand for the dopamine D2 receptor group, in the rat brain and spinal cord. J. Comp. Neurol. 344 (1), 121 https://doi.org/10.1002/cne.903440109.

Zai, Robbins, Sahakian, Kennedy, 2017. A review of molecular genetic studies of neurocognitive deficits in schizophrenia. Neurosci. Biobehav. Rev. 72, 50. https://doi org/10.1016/j.neubiorev.2016.10.024.

Zhang, Matos, Jego, Adamantidis, Séguéla, 2013. Norepinephrine drives persistent activity in prefrontal cortex via synergistic $\alpha 1$ and $\alpha 2$ adrenoceptors. PLoS One 8 (6), e66122.

Zitnik, 2016. Control of arousal through neuropeptide afferents of the locus coeruleus. Brain Res. 1641 (Pt B), 338. https://doi.org/10.1016/j.brainres.2015.12.010.

Zitnik, Curtis, Wood, Arner, Valentino, 2016. Adolescent social stress produces an enduring activation of the rat locus coeruleus and alters its coherence with the prefrontal cortex. Neuropsychopharmacology 41 (5), 1376. 